\title{
The plant bug genus Psallus (Heteropera: Miridae) in the Korean Peninsula with descriptions of three new species
}

\author{
Ram Keshari DUWAL ${ }^{1}$, Tomohide YASUNAGA², Sunghoon JUNG ${ }^{3}$ and Seunghwan LEE ${ }^{1 *}$ \\ ${ }^{1}$ Insect Biosystematics Laboratory, Research Institute for Agricultural and Life Sciences, Department of Agricultural Biotechnology, \\ Seoul National University, Seoul, 151-921, Korea; e-mail: phylinae@snu.ac.kr \\ ${ }^{2}$ Department of Entomology, Division of Invertebrate Zoology, American Museum of Natural History, New York, NY, 10024, USA; \\ e-mail: tyasunaga@amnh.org \\ ${ }^{3}$ Department of Entomology, University of California, CA 92521, USA; e-mail: sunghoon.jung@ucr.edu
}

Key words. Hemiptera, Heteroptera, Miridae, Psallus, new species, new records, Korean Peninsula

\begin{abstract}
There are twenty-six species of the genus Psallus Fieber, 1858 (Phylinae: Phylini) documented in the Korean Peninsula. Three new species are described: Psallus cheongtaensis sp. n., P. ernsti sp. n. and P. suwonanus sp. n., and four species are reported from Korea for the first time: Psallus cinnabarinus Kerzhner, 1979, P. flavescens Kerzhner, 1988, P. loginovae Kerzhner, 1988, and P. roseoguttatus Yasunaga \& Vinokurov, 2000. The genitalic structures of females and males are described and figured for most treated species. A key is provided for identifying all the Korean species.
\end{abstract}

\section{INTRODUCTION}

The genus Psallus (Miridae: Phylinae: Phylini) is a large Holarctic genus, comprising approximately 144 species (Schuh, 2003), most of which are recorded from temperate and cold temperate climatic zones, except for $P$. $(P$.$) buddha Yasunaga, which occurs in Thailand but is$ considered as a relic Palearctic element (Yasunaga, 2010). In eastern Asia, there are 44 species of Psallus (Kerzhner \& Josifov, 1999; Yasunaga \& Vinokurov, 2000) and the regional faunas have been documented from the Russian Far East (Kerzhner \& Josifov, 1966; Kerzhner, 1978, 1979, 1988a; Vinokurov, 1998), continental China (Zheng \& Li, 1990), the North Korea (Josifov, 1983, 1992) and Japan (Muramoto, 1973; Yasunaga \& Vinokurov, 2000).

Prior to this study, 19 species were known to occur in the Korean Peninsula (Kerzhner \& Josifov, 1999; Kwon et al., 2001). As a result of this study, 7 additional species are herein documented, and therefore, a total of 26 species are now confirmed to occur in this peninsula. Of these, three species (Psallus cheongtaensis sp. n., $P$. ernsti sp. n. and P. suwonanus sp. n.) are described as new, and the following four species are reported for the first time in the Korean Peninsula: $P$. cinnabarinus Kerzhner, 1979, P. flavescens Kerzhner, 1988, P. loginovae Kerzhner, 1988 and $P$. roseoguttatus Yasunaga \& Vinokurov, 2000.

Psallus is often referred as a "garbage genus" (Yasunaga \& Vinokurov, 2000), based primarily on the similarity in their external appearances with that of species in other phyline groups. Many authors have provided consistent definitions for the genus (Fieber, 1858, 1861; Reuter, 1883, 1899; Wagner, 1952, 1970, 1975; Wagner
\& Weber, 1964; Kerzhner, 1962; Yasunaga \& Vinokurov, 2000, etc.). However, their classification is still unsatisfactory and the genus requires a stable diagnosis. Psallus is currently subdivided into eight subgenera (Kerzhner \& Josifov, 1999; Yasunaga \& Vinokurov, 2000) mainly on the basis of similarity in the male genitalia: Apocremnus Fieber, 1858, Calopsallus Yasunaga \& Vinokurov, 2000, Hylopsallus Wagner, 1952, Mesopsallus Wagner, 1970, Phylidea Reuter, 1899, Pityopsallus Wagner, 1952, Subpsallus Linnavuori, 1993 and a nominotypical subgenus.

Based on our examination of female genitalia the subgeneric placement of some Korean species remains doubtful. As only species from Korea and the adjacent regions of eastern Asia are included in this study the placement of such doubtful members is beyond the scope of the present study. The objective of this paper is to clarify the basic faunal composition and biodiversity of the genus Psallus in the Korean Peninsula and to provide a positive diagnosis for each species.

The female genitalia have been insufficiently used for classifying the Phylini. In this study, the female and male genitalia of each possible species were studied and described. A key is provided to identify all Psallus species in Korea.

\section{MATERIAL AND METHODS}

About 400 specimens were examined during the course of this study. Most of them were collected by the first and third authors between May 2008 and June 2011 and are preserved in the Biosystematics Laboratory, Seoul National University, South Korea (SNU). Additional material from the following research institutes and personal collections were also examined: National Academy of Agricultural Science, South Korea (NAAS); the

\footnotetext{
* Corresponding author; e-mail: seung@snu.ac.kr
} 
private North Korean collection (ex M. Josifov) of E. Heiss, the Tiroler Landesmuseum Ferdinandeum, Innsbruck, Austria (TLMF); T. Yasunaga's collection, Nagasaki, Japan (TYCN) and a few related specimens collected by I.M. Kerzhner held in the Zoological Institute, Russian Academy of Sciences, Laboratory of Insect Taxonomy, St. Petersburg, Russia (ZISP). Specimens examined are retained in the above collections as indicated in the text by their respective institutional acronyms.

Matrix code labels that uniquely identify each specimen were attached to type material and some representative specimens of known species, and are referred to as "unique specimen identifiers" (USIs). Generally each USI label corresponds to a single specimen; however, some USI labels correspond to 2-3 specimens when several specimens are mounted on one pin. More information on these specimens is available on the PBI Project on Plant Bugs (http://research.amnh.org/pbi/ heteropteraspeciespage/) and "www.discoverlife.org" websites.

Habitus photographs in the figures were taken through the objective of a Leica DE/S8 APO microscope using a camera model: "14.2 color mosaic". All the illustrations of genitalia were prepared using a Leica DE/DM 4000B microscope.

All measurements are in millimeters. The terminology of male genitalia mainly follows Cassis (2008), and that of females, Davis (1955), Schuh (2006), Schuh \& Wu (2009), Wyniger (2006), and Yasunaga \& Schwartz (2007). Each illustration of female genitalia shows the bursa copulatrix in dorsal view. New distributional records for known species are indicated by an asterisk $\left(^{*}\right)$ after the locality name. Only selected references are cited in the synonymic list of the known taxa because comprehensive catalogues are now available (Wheeler \& Henry, 1992; Schuh, 1995; Kerzhner \& Josifov, 1999).

\section{SYSTEMATICS}

\section{Genus Psallus Fieber, 1858}

Psallus Fieber, 1858: 321; Schuh, 1995: 397 (cat.); Kerzhner \& Josifov, 1999: 399 (cat.); Yasunaga \& Vinokurov, 2000: 653 (diag.); Yasunaga, 2001: 171 (diag.); Kwon et al., 2001: 177 (cat.).

Type species. Lygaeus sanguineus Fabricius, 1794 (= Cimex haematodes Gmelin, 1790), subsequent designation by Reuter, 1888: 412.

Diagnosis. Male: Body varying in colour from uniformly yellow, orange, red, pale or dark brown, to black, sometimes variously spotted or mottled, rarely tinged with green; body shape oval to elongate oval and 2.9 to 5.5 long; dorsal surface with uniformly distributed, simple setae and with silvery scale-like setae that are often easily abraded; dorsal surface of head, pronotum, scutellum and hemelytra more or less shagreened; metafemora incrassate, tumid; endosoma C- or J-shaped, with a complex apical section; secondary gonopore located medially or sub-apically.

Female: Generally similar to male, but body usually shorter, more oval and sometimes paler in coloration. Bursa copulatrix variable in size; dorsal labiate plate mostly furnished with clusters of spinules laterally and with variously folded membranes; sclerotized rings distinct, variable in shape, small or large, and/or oval, elongate oval or circular.

Distribution. Holarctic Region and Central Thailand.

Biology. Most members of Psallus are associated with broadleaf host plants. Some species are serious agricul- tural pests (e.g., P. ambiguus is a vector of stony pit disease in pears) (Taksdal, 1983) and immatures of the same species also feed on animal food (Wheeler, 2001). Zoophagy was confirmed by the first author who observed nymphs of $P$. ater feeding on coleopteran and lepidopteran larvae during laboratory tests. Some members of this genus are occasionally attracted to light.

Discussion. Psallus is the largest genus in the tribe Phylini and is a difficult group to define. Seven of the eight subgenera (except subgenus Subpsallus) occur in the Korean Peninsula and most of them are common in temperate and cold temperate zones of neighbouring countries, China, Japan and the Russian Far East.

The female genitalia of all the possible members found in Korea during this study were examined. It was observed that females of the subgenera Calopsallus $[P$. (C.) roseoguttatus, $P$. (C.) clarus, $P$. (C.) tesongsanicus, Figs 14D-E, 15A] and Pityopsallus $[P$. (P.) vittatus, Fig. $15 \mathrm{E}]$ have genitalia structures distinctly different from those of other five subgenera which all have a similar basic structure [e.g., P. (Apocremnus) michaili, Fig. 14C; P. (Hylopsallus) tonnaichanus, Fig. 15B; P. (Mesopsallus) samdzijonicus, Fig. 15D; P. (Phylidea) castaneae, Fig. 13D; and P. (Psallus) amoenus, Fig. 13B]. This makes it difficult to define their sub-generic status.

The three new species described here are placed in three subgenera of Psallus: P. (Hylopsallus) suwonanus, P. (Phylidea) ernsti and P. (Psallus) cheongtaensis, based on the similarity of their endosoma with respective members.

\section{Key to the Korean species of Psallus}

1 Antennal segment II pale, yellow, or brown ......... 2

- Antennal segment II entirely dark or black, or, either base or apex or both black. . . . . . . . . . . . . . 21

2 Antennal segment I pale or with dark base . . . . . . . . 3

- Antennal segment I entirely black . . . . . . . . . . . . 22

3 Dorsum variously coloured, without speckles ........ 7

Dorsum pale with red or orange speckles . . . . . . . . 4

4 Head without brown spots; anterior pronotum with numerous brown or orange red spots (Fig. $8 \mathrm{~F}-\mathrm{G}$ ). Male genitalia (Figs 12A-C): Endosoma C-shaped, secondary elongated process extends through the blunt apex (Josifov, 1983: 204); right paramere with tooth-like out growth near the apex. Female genitalia (Fig. 13B): Dorsal labiate plate with rectangular membranous folds posteriorly and furnished with numerous spines around sclerotized rings. . . . . . ................................. P. amoenus Head and pronotum with brown spots . . . . . . . . . 5

5 Mesoscutum and scutellum densely covered with brown spots but without speckles; cuneus with few speckles or speckles only at base (Fig. 4C-D). Male genitalia (Figs 5A-E): Endosoma C-shaped, apex blunt with numerous spinules and subapical elongated secondary process short; right paramere apically extended into an elongated protuberance. Female genitalia (Fig. 14D): Sclerotized rings very small, lateral oviducts posteriorly supported by bowl shaped membranous folds, cluster of spinules on dorsal labiate plate located far from sclerotized rings.............. P. clarus - Mesoscutum and scutellum with only red or orange speckles . . . . . . . . . . . . . . . . . . . . . . 6 
6 Femora pale, apically tinged with red, covered with densely distributed large and small fused spots and dorsally a few spots on sub-apical region (Fig. 4A-B). Male genitalia (Figs 3J-M): Endosoma C-shaped, apex broad and furnished with spinules, elongated secondary process furnished with tooth like spines on inner margin. Female genitalia (Fig. 14E): Sclerotized rings comparatively small, lateral oviducts positioned within approximately heart shaped folds, posterior portion of dorsal labiate plates wide and laterally furnished with spinules. . . . . . . . . . . . . . . . Poseoguttatus Femora pale ventrally with numerous small spots on distal half and dorsally with few spots sub-apically (Fig. 4E-F). Male genitalia (Figs 5F-J): Endosoma J-shaped, broad, apex with several short and blunt processes, with spines on margins, one of the processes somewhat elongated and with only one spine apically. Female genitalia (Fig. 15A): Sclerotized rings ovate but posterior margin straight, lateral oviduct supported by membranous folds...... P. tesongsanicus

7 Dorsum dark, brown or black or castaneous . . . . . . . 8 8

- Dorsum pale or with various bright colours . . . . . . . 10

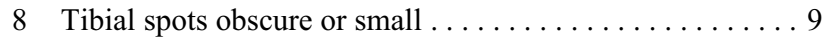

- Tibial spots large ...................... 16

9 Dorsum dark brown or black, in brownish individuals head, pronotum and scutellum shagreened and blackish; femora dark with rows of dark brown spots on distal half, tibia pale brown with small obscure spots at the base of black spines (Fig. 10B-C). Male genitalia (Figs 11K-O): Endosoma nearly s-shaped, apex extends to form elongated process furnished with toothed spinules on outer margin; Female genitalia (Fig. 15E): Structures delicate, with a socket like membranous folds................. P. vittatus

Dorsum brown; head, pronotum, mesoscutum and scutellum dark brown or blackish; femora pale brown, ventrally with dark brown spots on apical half, tibiae with small brown spots at the base of brown spines (Fig. 10A). Male genitalia (Figs $11 \mathrm{~F}-\mathrm{J}$ ): Endosoma nearly s-shaped, apex extends to form elongated process, furnished with spinules on the basal half of outer margin, and a membrane reaching half the length of endosoma from apex, and with a serrated lateral margin. ............................ P. luridus

10 Body red or orange or orange red $\ldots \ldots \ldots \ldots \ldots \ldots 11$

- Body uniformly pale (or somewhat pale yellowish), genital segment with a pair of protuberances on either side (Fig. $10 \mathrm{H}$ ). Male genitalia (Figs 12H-N): Endosoma C-shaped, apex with a pair of small ear like processes laterally and an elongated flat apical process medially.

P. cheongtaensis sp $\mathrm{n}$

11 Body uniformly orange (Fig. 8B). Male genitalia (Figs $9 \mathrm{~K}-\mathrm{N}$ ): Endosoma J-shaped, apical elongated process on apex slightly curved inwards, membranous outgrowth with few spinules basally, secondary gonopore located apically. Female genitalia (Fig. 14A): Sclerotized rings small, oval, somewhat straight posteriorly and pointed anteriorly, membranous folds on dorsal labiate plate furnished with dense spinules laterally ................... flavescens Body other than orange coloured. . . . . . . . . . . 12

12 Hemelytra deeply red . . . . . . . . . . . . . . . . . 13 Corium brownish red . . . . . . . . . . . . . . . 14

13 Head, pronotum, mesoscutum, scutellum castaneous; femora with apical $2 / 3$ red and with few spots on anterior margin (Fig. 8A). Male genitalia (Figs 9F-J): Endosoma J-shaped, margin of apex with spinules and a short apical outgrowth. Female genitalia (Fig. 13E): Sclerotized ring small, oval pointed anteriorly, membranous folds on dorsal labiate plate medially invaginated. . . . . . . . . . P. cinnabarinus
- Head with pale vertex; pronotum, mesoscutum, scutellum and the base of hemelytra blackish, and shiny (dark red specimen, male) or orange red except for the dark callus (faintly red in female) (Fig. 10I-J); metafemora with black spots along the entire anterior margin ....... P. koreanus

14 Anterior margin of pronotum or calli with a large black spot; vertex and base of cuneus pale; corium and femora red (dark red specimens), or posterior part of pronotum, apex of scutellum and base of corium pale with larger posterior part dark tinged with red, and femora pale (pale specimens) (Fig. $8 \mathrm{H}-\mathrm{K})$ with two rows of spots proximally, which distally merge irregularly...................... P. ulmi

- Pronotum reddish brown, or pale orange, or same as colour as hemelytra. . . . . . . . . . . . . . . 15

15 Body pale, dorsum pale tinged with orange yellow or pale brown, head yellow to dark brown; apex of clavus slightly darker, metafemora with distinctly large and small spots on distal half, membrane brownish (Fig. 10D-E). . . . . P. kimi

- Head ocher, fore head with dark markings, clypeus black brown. . . . . . . . . . . . . . . . . . P. sanguinarius

16 Genital segment with tuft of stiff hairs laterally. Male genitalia (Figs 7A-E): Endosoma J-shaped, apex extended into an elongated process, at the base of which there are membranous folds furnished with spinules. Female genitalia (Fig. 15B): Sclerotized rings broad and somewhat sub-rectangular, dorsal labiate plate somewhat sclerotized, membranous folds arise between sclerotized rings. . P. tonnaichanus - Genital segment without a tuft of stiff hairs . . . . . . . 17

17 All femora black, apically tinged with red except for pale apex of fore femur. Male genitalia (Figs 9A-E): Endosoma J-shaped, sub-apically extended membranous folds covered with numerous spinules. Female genitalia (Fig. 13D): Sclerotized ring elongated, width narrow, membranous folds located centrally..................... castaneae - Femora castaneous, or apically pale (in all), or base of femora black and apex red ................. 18

18 Labium reaches apex of mesocoxa; base of femora black, $1 / 2$ or $3 / 4$ from apex reddish and covered with black spots (Fig. 4G-H). Male genitalia (Figs 7F-J): Endosoma C-shaped, short apical process with cluster of spinules. Female genitalia (Fig. 15C): Sclerotized rings elongated oval, posterior portion of dorsal labiate plate with inverted V-shaped membranous folds......... P. suwonanus $\mathrm{sp} . \mathrm{n}$. - Labium reaches metacoxae or at least exceeds mesocoxae . . ................................ 19

19 Body small about 2.9-3.0; spots on metatibiae castaneous (Fig. 6G-H). Male genitalia (Figs 9O-T): Endosoma nearly S-shaped, apical margin completely surrounded by spinules and elongate process slender, base projecting from secondary gonopore, which is positioned near apex. Female genitalia (Fig. 15F): Sclerotized rings small, ovate, somewhat pointed anteriorly.................. P. ernsti sp. n.

- Body medium sized, spots on tibia brown or dark brown at the base of black spines . . . . . . . . . . . . 20

20 Coxae dark brown, trochanters pale .......... P. kerzhneri - Coxae black, trochanters dark brown to blackish. Male genitalia (Figs 11A-E): Endosoma J-shaped, apically ending in a leaf like structure. Female genitalia (Fig. 14F): Sclerotized rings are strongly asymmetrical. .........P. loginovae

21 All antennal segments black. Male genitalia (Figs 3E-I): Endosoma nearly S-shaped, apical region membranous with numerous clusters of spinules and the outgrowing process short and slender. Female genitalia (Fig. 14C): Sclerotized rings distinct, vase shaped membranous folds at posterior portion of dorsal labiate plate. ............ P. michaili Antennal segments either with base or apex or both black 22 

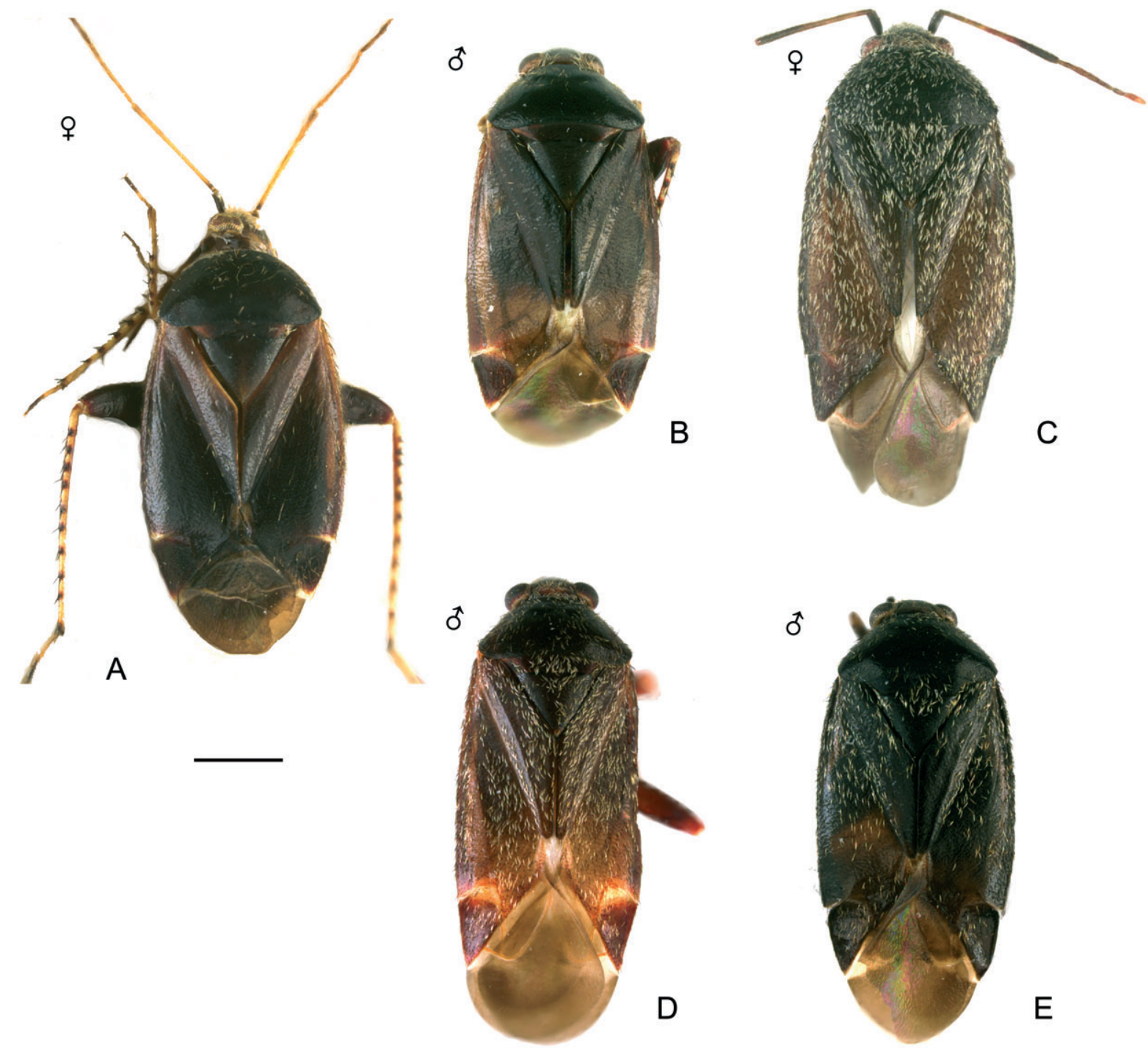

Fig. 1. Habitus images of Psallus spp. A-B - P. ater; C - P. atratus; D - P. betuleti; E - P. aethiops. Scale bar: $0.5 \mathrm{~mm}$.

22 Male blackish, female brownish; head, pronotum, and scutellum black, in female with pale medial stripe extended from fore head to pronotum, which is enlarged as a spot in each medial region, scutellum with 3 pale spots and apex of scutellum pale; metafemora pale with chain of spots joining each other, tibia with large castaneous spots at the base of pale spines. Male genitalia (Figs 12D-G): Endosoma roughly s-shaped, extended outgrowth process appears as a horny spine together with other large spines arising from apex. Female genitalia (Fig. 13C): Sclerotized ring small, oval, broad posteriorly and narrow anteriorly, posterior portion of dorsal labiate plate with membranous folds supporting lateral sclerotized ring.......... P. bagjonicus

- Head, pronotum and scutellum without spots, femur casta-

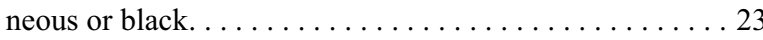

23 Largest Korean species 4.8-5.5, vertex and inner margin of eyes pale, head and pronotum black, hemelytra and legs blackish brown (Fig. 6C-D). Female genitalia (Fig. 15D): Sclerotized rings elongate oval, with apex extended in the form of a protuberance that reaches spinules on dorsal lateral plates ......................... P. samdzijonicus

- Size smaller, vertex pale or black, if pale only at central margin, femora black or castaneous and tibiae pale .... 24

24 Ostiolar region greyish or pale . . . . . . . . . . . 25
- Ostiolar region black ..................... 26

25 Cuneus castaneous red with pale base, tibiae pale tinged with red and with small castaneous spots (Fig. 1D). Male genitalia (Figs 3A-D): Endosoma nearly S-shaped, laterally furnished with spinules sub-apically, and elongated apical process slender and slightly curved opposite of spinules .... P. betuleti

- Cuneus black (or castaneous), apically tinged with dark red, tibiae pale with large castaneous red spots. Male genitalia (Figs 2D-H): Endosoma C-shaped, simple apex without spinules, apical process short and slender. Female genitalia (Fig. 13A): dorsal labiate plate with series of spinules laterally; posterior portion of dorsal labiate plate with numerous membranous folds; sclerotized rings elongated and tapered

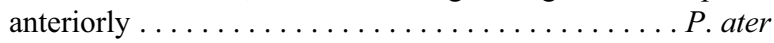

26 Fore and meso femora apically pale, tibiae with small brown spots. Male genitalia (Figs 2A-C): Endosoma nearly S-shaped, membranous folds arise from apex of secondary gonopore and bear several spinules sub-apically. P. aethiops

- Femora entirely black and shiny, tibiae with large castaneous black spots; male genitalia (Figs 2I-M): Endosoma C-shaped, membranous folds arise from apex of secondary gonopore and are densely furnished with spinules laterally and sparsely towards middle .............. P. atratus 

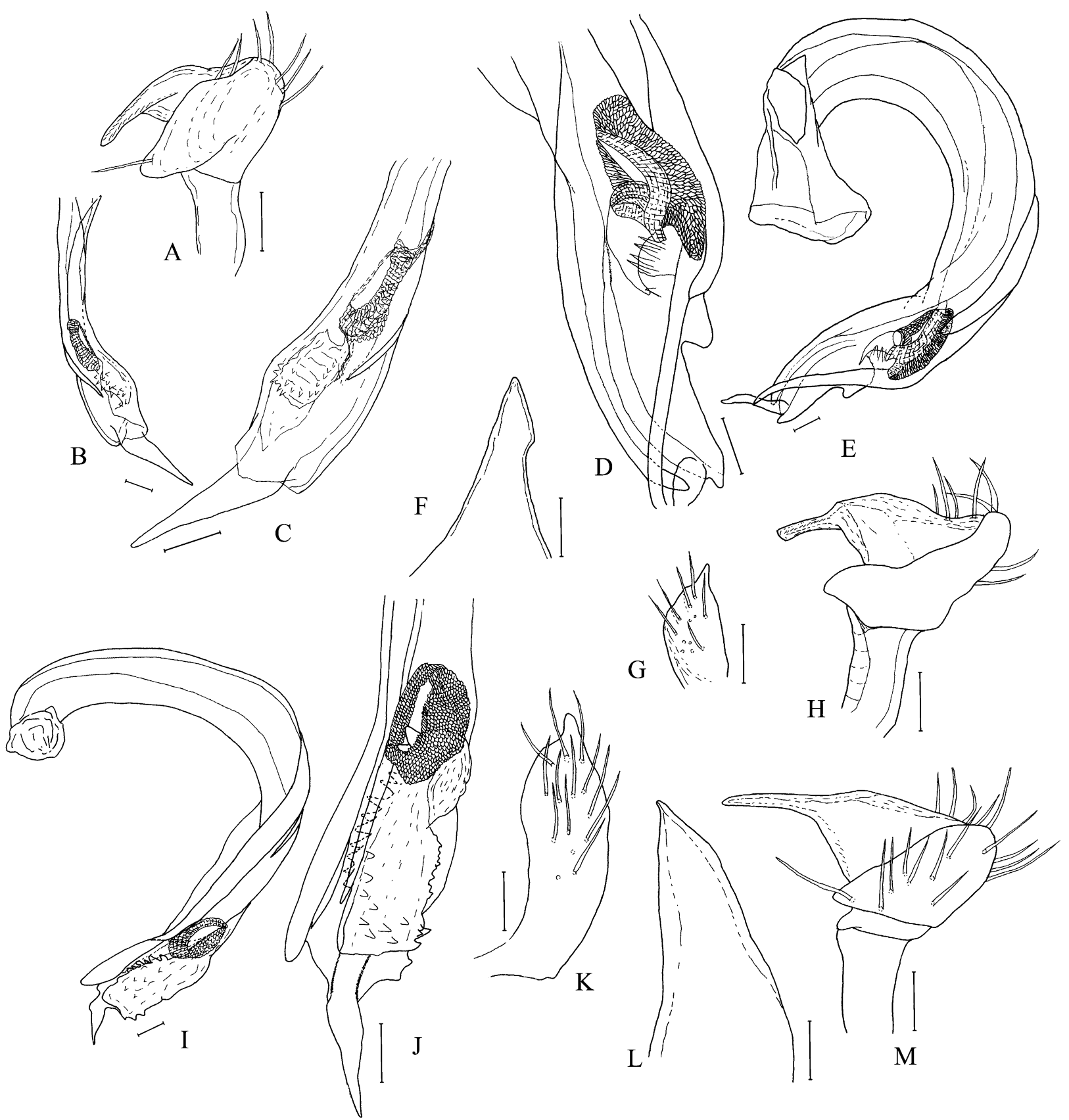

Fig. 2. Male genitalia of Psallus spp. A-C - P. aethiops; D-H - P. ater; I-M - P. atratus. A, H, M - left paramere; G, $\mathrm{K}-$ right paramere; B-E, I-J - endosoma; F, L - phallotheca. Scale bars: $0.1 \mathrm{~mm}$.

\section{Subgenus Apocremnus Fieber, 1858}

The genus Apocremnus Fieber was downgraded to subgenus Psallus by Reuter $(1895,1878)$ and includes fifteen species (Kerzhner \& Josifov, 1999; Yasunaga \& Vinokurov, 2000).

Species of this subgenus are mostly distinguished by darker body coloration, pale or dark appendages; C- or S-shaped endosoma with a membranous structure arising either mesially or from the apex of the secondary gonopore and furnished with minute spinules (Figs 2B-C, D-E, I-J; 3C-D, E-F). For a detail description see Wagner, 1975.

\section{Psallus (Apocremnus) aethiops (Zetterstedt, 1838)}

Figs $2 \mathrm{~A}-\mathrm{C}$

Phytocoris aethiops Zetterstedt, 1838: 274.

Psallus aethiops: Kulik, 1965: 62 (list); Kerzhner, 1973: 91 (list); 1988a: 845 (key, fig.); Schwartz \& Kelton, 1990: 945 (diag., desc.); Wheeler \& Henry, 1992: 180 (distr., host); Schuh, 1995: 399 (cat.); Kerzhner \& Josifov, 1999: 400 (cat.); Anufriev et al., 2001: 124 (Engl. transl.); Kwon et al., 2001: 177 (cat.).

Diagnosis. Entirely black body, pale basal margin of vertex, completely black antennal segments I and II, dark ostiolar peritreme, black femora with pale apices, and pale or pale brown tibiae with brown base and dark 


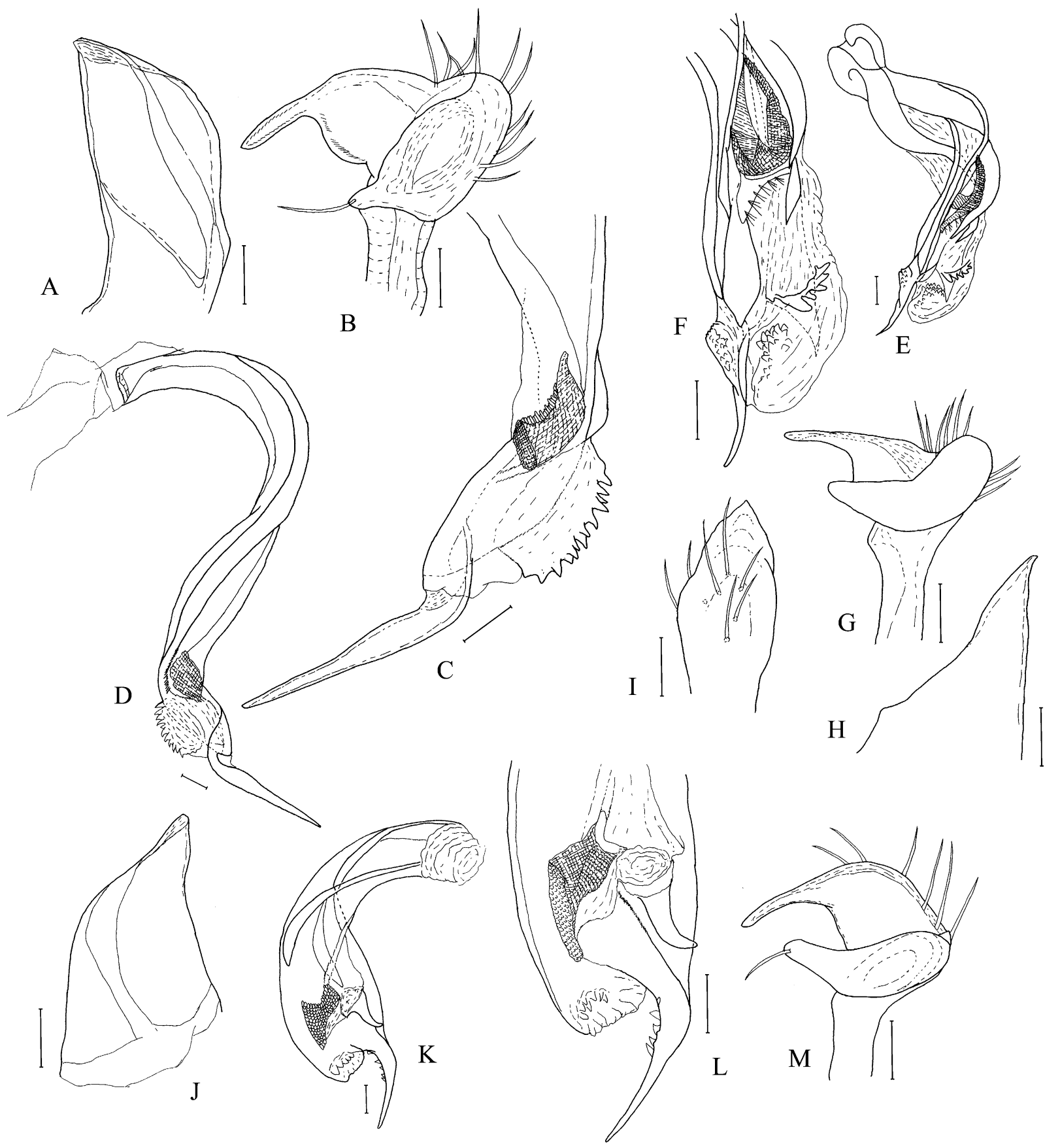

Fig. 3. Male genitalia of Psallus spp. A-D - P. betuleti; E-I - P. michaili; J-M - P. roseoguttatus. B, G, M - left paramere; Iright paramere; C-F, K-L - endosoma; A, H, J-phallotheca. Scale bars: $0.1 \mathrm{~mm}$.

brown spots at the base of black spine. For a detail description see Schwartz \& Kelton, 1990: 945.

Male genitalia (Figs 2A-C): Endosoma nearly $\mathrm{S}$-shaped, slender and apically prolonged in the form of a flat apical process, membranous structure arises from apex of secondary gonopore and furnished with several spinules subapically (Figs $2 \mathrm{~B}-\mathrm{C}$ ). Left paramere as illustrated in Fig. 2A.

Specimens examined. Russia: 10 , S. Yakutia, $18 \mathrm{~km} \mathrm{NE}$ of Kolyma River, 577 m alt., 13.vii.1995, on Salix sp. (Salicaceae), T. Yasunaga, determined by T. Yasunaga, 2000 (NAAS).
Distribution. China, Korea (North), Russia (Far East, Tuva, Siberia), Europe, Canada, USA (Alaska, Michigan, Pennsylvania).

Biology. Kerzhner (1988a) documented the host plant of this species as willow and the specimen in this study was also collected by Yasunaga on Salix sp. (Salicaceae).

\section{Psallus (Apocremnus) ater Josifov, 1983}

Figs 1A-B, 2D-H, 13A, 17A

Psallus (Apocremnus) ater Josifov, 1983: 198 (desc., figs); 1992a: 116 (list); Schuh, 1995: 401 (cat.); Kerzhner \& Josifov, 1999: 401 (cat.); Kwon et al., 2001: 177 (cat.). 
TABLE 1. Measurements (in mm) of Psallus spp. from Korea. $\mathrm{M}$ - male, $\mathrm{F}$ - female, $\mathrm{N}$ - number of specimens measured, and SD - standard deviation. Specimens of the newly described species and those collected during the field survey in South Korea were measured.

\begin{tabular}{|c|c|c|c|c|c|c|c|c|c|c|c|c|c|c|c|c|}
\hline \multirow{4}{*}{ P. ater } & \multirow{8}{*}{$\begin{array}{l}\text { Mean } \\
\text { SD } \\
\text { Min } \\
\text { Max }\end{array}$} & \multicolumn{11}{|c|}{ Length } & \multicolumn{4}{|c|}{ Width } \\
\hline & & \multirow{2}{*}{$\begin{array}{l}\text { Total } \\
\text { body }\end{array}$} & \multirow{2}{*}{ Head } & \multirow{2}{*}{ Labium } & \multirow{2}{*}{$\begin{array}{c}\text { Pro- } \\
\text { notum }\end{array}$} & \multicolumn{4}{|c|}{ Antenna } & \multicolumn{3}{|c|}{ Hind leg } & \multirow{2}{*}{ Head } & & Pro- & \\
\hline & & & & & & Seg. I & Seg. I & I Seg. & II Seg. IV & Femur & Tibia & Tarsus & & & & lyt \\
\hline & & & & & & & & & & & & & & & & \\
\hline $\mathrm{M}(\mathrm{N}=5)$ & & 3.47 & 0.55 & 1.39 & 0.71 & 0.21 & 1.04 & 0.57 & 0.37 & 1.27 & 2.1 & 0.52 & 0.79 & 0.37 & 1.38 & 1.7 \\
\hline & & 0.027 & & & 0.03 & 0.03 & 0.09 & 0.01 & 0.03 & 0.13 & 0.13 & & 0.03 & 0.03 & 0.07 & 0.1 \\
\hline & & 3.21 & 0.44 & & 0.65 & 0.17 & 0.89 & 0.55 & 0.34 & 1.09 & 1.92 & 0.45 & 0.75 & 0.35 & 1.26 & 1.58 \\
\hline & & 3.86 & 0.61 & & 0.74 & 0.25 & 1.12 & 0.58 & 0.4 & 1.41 & 2.24 & & 0.83 & 0.41 & 1.48 & 1.82 \\
\hline P. atratus & & & & & & & & & & & & & & & & \\
\hline $\mathrm{F}(\mathrm{N}=1)$ & Mean & 4.39 & 0.68 & 1.61 & 0.84 & 0.2 & 1.16 & 0.6 & 0.36 & 1.45 & 2.14 & 0.58 & 0.89 & 0.45 & 1.61 & 1.99 \\
\hline P. bagjonic & & & & & & & & & & & & & & & & \\
\hline $\mathrm{M}(\mathrm{N}=1)$ & Mean & 3.52 & 0.56 & & 0.68 & 0.2 & 1.07 & 0.45 & 0.28 & 1.08 & 1.7 & & 0.83 & 0.37 & 1.31 & 1.52 \\
\hline $\mathrm{F}(\mathrm{N}=5)$ & Mean & 3.3 & 0.56 & 1.23 & 0.67 & 0.19 & 0.89 & 0.38 & 0.24 & 1.09 & 1.68 & 0.39 & 0.76 & 0.39 & 1.36 & 1.75 \\
\hline & SD & 0.24 & 0.04 & & 0.02 & 0.04 & 0.04 & 0 & 0.0 & 0.02 & 0.02 & & 0.02 & 0.01 & 0.04 & 0.08 \\
\hline & Min & 3.01 & 0.51 & 1.2 & 0.66 & 0.1 & 0.84 & 0.38 & 0.2 & 1.07 & 1.66 & 0.34 & 0.73 & 0.38 & 1.31 & 1.64 \\
\hline & & & 0.61 & & 0.7 & & 0.93 & & & & 1.2 & & 0.8 & 0.41 & 1.41 & 1.82 \\
\hline P.cas & & & & & & & & & & & & & & & & \\
\hline & Mean & 3.16 & 0.55 & 1.14 & 0.66 & 0.21 & 0.88 & 0.47 & 0.32 & 1.1 & 1.76 & 0.45 & 0.77 & 0.36 & 1.32 & 1.59 \\
\hline & SD & & 0.03 & & 0.02 & & 0.04 & & & & 0.07 & & 0.02 & 0.02 & 0.05 & 2 \\
\hline & Min & 2.99 & 0.5 & & 0.63 & 0.2 & 0.83 & & & & 0.1 & & 0.07 & 0.34 & 1.26 & 1.57 \\
\hline & Max & 3.37 & 0.57 & & 0.7 & & 0.93 & & & 4 & 1.81 & & 0.79 & 0.39 & 1.37 & 1.62 \\
\hline $\mathrm{F}(\mathrm{N}=5)$ & Mean & 3.21 & 0.52 & & 0.66 & 0.3 & 0.89 & & 03 & 13 & 1.69 & & 0.74 & 0.39 & 1.32 & 1.6 \\
\hline & $\mathrm{SD}$ & & 0.03 & & 0.02 & & 0.05 & 0.02 & & & 0.02 & & 0.01 & 0.01 & 0.04 & 0.04 \\
\hline & & & 0.49 & & 0.63 & & 0.82 & 0.51 & 0.2 & & 1.6 & & 0.72 & 0.39 & 1.2 & 4 \\
\hline & Max & 3.42 & 0.57 & & 0.69 & 1.1 & 0.95 & & & & 1.7 & & 0.76 & 0.41 & 1.37 & 1.63 \\
\hline & & n. & & & & & & & & & & & & & & \\
\hline $\mathrm{M}(\mathrm{N}=1)$ & MeaI & 3.4 & 0.48 & 1.44 & 0.66 & 0.21 & 1.01 & 0.46 & 0.37 & 1.25 & 1.8 & 0.46 & 0.85 & 0.37 & 1.27 & 1.55 \\
\hline P. cinnaba & inus & & & & & & & & & & & & & & & \\
\hline $\mathrm{M}(\mathrm{N}=5)$ & ean & 3.06 & 0.52 & & 0.6 & & 1.07 & & & & & & 0.78 & & 1.14 & 1.44 \\
\hline & SL & & & & 0.05 & & 0.05 & & & & & & & 1 & 0.0 & \\
\hline & Min & 2.99 & 0.48 & & 0.52 & 0.2 & 1 & 0.5 & 0.3 & 94 & 1.8 & 9 & 0.75 & 0.31 & 1.0 & 1.38 \\
\hline & & 3.19 & 0.58 & 1. & 0.64 & 0.24 & 1.13 & 0.71 & 03 & 1.22 & 2.02 & 0.4 & 0.81 & 0.35 & 1.22 & 1.49 \\
\hline $\mathrm{F}(\mathrm{N}=1)$ & & 3.54 & 0.53 & & 0.68 & 0.22 & 1.11 & 0.65 & 0.38 & 1.13 & 2.01 & 0.45 & 0.77 & 0.46 & 1.21 & 1.67 \\
\hline P. clarus & & & & & & & & & & & & & & & & \\
\hline $\mathrm{M}(\mathrm{N}=5)$ & Mean & 3.99 & 0.62 & 1.48 & 0.73 & 0.29 & 1.23 & 0.59 & 0.29 & & 2.21 & & 0.87 & 0.34 & 1.52 & 1.8 \\
\hline & SD & & 0.02 & & 0.02 & & 0.04 & & & & 0.0 & & 0.01 & 0.02 & 0.02 & 0.1 \\
\hline & & & 0.6 & & 0.7 & & 1.17 & & & 27 & & & 0.85 & 0.32 & 1.5 & 1.67 \\
\hline & $\mathrm{Ma}$ & 4.17 & 0.65 & & 0.75 & & 1.27 & & & & 2.3 & & 0.89 & 0.36 & 1.55 & 1.96 \\
\hline $\mathrm{F}(\mathrm{N}=5)$ & Mea & & & & 0.72 & & 1.2 & & & & & & & & & \\
\hline & SI & & 0.04 & & 0.05 & & 0.05 & & & & & & & & & \\
\hline & Min & & 0.6 & & 0.68 & 0.22 & 1.15 & 0.5 & 0.3 & 29 & 2.08 & 0.4 & 0.84 & 0.37 & 1.51 & 1.7 \\
\hline & Max & & 0.69 & & 0.8 & 0.24 & 1.28 & 0.62 & 03 & & 2.3 & & 0.93 & 0.43 & 1.64 & 1.91 \\
\hline & & & & & & & & & & & & & & & & \\
\hline & & & & & & & & & & & & & & & 1.21 & \\
\hline & SD & & 0.0 & & 0.03 & & 04 & & & & & & & & 0.0 & 99 \\
\hline & Min & 2.61 & 0.45 & & 0.55 & 0.13 & 0.7 & 0.37 & 0.25 & 0.67 & 1.29 & 0.3 & 0.72 & 0.36 & 1.18 & 1.38 \\
\hline & Max & 3.22 & 0.53 & & 0.62 & & 0.74 & 0.42 & 0.3 & 0.76 & 1.34 & & 0.76 & 0.39 & 1.22 & 1.55 \\
\hline $\mathrm{F}(\mathrm{N}=5)$ & Mean & 3 & 0.5 & & 0.6 & & 0.68 & & & & 1.39 & & 0.74 & 0.37 & & 1.51 \\
\hline & SI & & & & & & & & & & & & & & 0.0 & \\
\hline & Min & & & & 0.55 & & 0.61 & & & & & & & & & \\
\hline & Max & 3.12 & 0.55 & & 0.66 & 0.16 & 0.76 & 0.41 & 0.3 & 0.69 & 1.48 & 0.42 & 0.79 & 0.42 & 1.28 & 1.72 \\
\hline & & & & & & & & & & & & & & & & \\
\hline & Mean & & 0.5 & & 0.56 & & 1.08 & & & & & & 0.75 & & 1.15 & 1.42 \\
\hline & SD & & & & & & & & & & & & & 0.002 & & \\
\hline & Min & 3.39 & 0.48 & & 0.55 & 0.23 & 1 & 0.43 & 0.36 & & 2 & & 0.74 & 0.32 & & 1.39 \\
\hline & Max & 3.42 & 0.51 & 1.1 & 0.57 & 0.24 & 1.16 & 0.6 & 0.38 & 1.27 & 2.03 & 0.53 & 0.76 & 0.32 & 1.2 & 1.46 \\
\hline $\mathrm{F}(\mathrm{N}=4)$ & Mean & 3.35 & 0.56 & 1.42 & 0.54 & 0.22 & 1.1 & 0.57 & 0.38 & & 1.99 & 0.41 & 0.71 & 0.36 & 1.18 & 1.53 \\
\hline & SD & 0.23 & 0.02 & 0.38 & 0.05 & 0.03 & 0.01 & 0.01 & 0.03 & 0.07 & 0.03 & 0.07 & 0.02 & 0.01 & 0.04 & 0.62 \\
\hline & Min & & 0.54 & 1.2 & 0.51 & & 1.08 & 0.56 & 0.36 & & 1.97 & & 0.7 & 0.35 & 1.13 & 1.46 \\
\hline & Max & 3.66 & 0.59 & 1.99 & 0.61 & 0.24 & 1.11 & 0.57 & 0.4 & 1.28 & 2.01 & 0.46 & 0.74 & 0.37 & 1.23 & 1.59 \\
\hline
\end{tabular}


TABLE 1 (continued).

\begin{tabular}{|c|c|c|c|c|c|c|c|c|c|c|c|c|c|c|c|c|}
\hline & \multicolumn{11}{|c|}{ Length } & \multicolumn{4}{|c|}{ Width } \\
\hline & & \multirow{2}{*}{$\begin{array}{l}\text { Total } \\
\text { body }\end{array}$} & \multirow{2}{*}{ Head } & \multirow{2}{*}{ Labium } & \multirow{2}{*}{$\begin{array}{c}\text { Pro- } \\
\text { notum }\end{array}$} & \multicolumn{4}{|c|}{ Antenna } & \multicolumn{3}{|c|}{ Hind leg } & \multirow{2}{*}{ Head } & \multirow{2}{*}{ Vertex } & \multirow{2}{*}{$\begin{array}{l}\text { Pro- } \\
\text { notum }\end{array}$} & \multirow{2}{*}{$\begin{array}{l}\text { Hem- } \\
\text { elytra }\end{array}$} \\
\hline & & & & & & $\overline{\text { Seg. I }}$ & Seg. 1 & II Seg. I & $\overline{\text { IIISeg. IV }}$ & Femur & Tibia & Tarsus & & & & \\
\hline \multicolumn{17}{|c|}{ P. loginovae } \\
\hline \multirow[t]{4}{*}{$\mathrm{M}(\mathrm{N}=6)$} & Mean & 3.36 & 0.56 & 1.29 & 0.7 & 0.22 & 0.93 & 0.55 & 0.32 & 1.22 & 1.88 & 0.48 & 0.78 & 0.39 & 1.38 & 1.64 \\
\hline & $\mathrm{SD}$ & 0.26 & 0.03 & 0.08 & 0.02 & 0.04 & 0.04 & 0.02 & 0.03 & 0.06 & 0.06 & 0.05 & 0.01 & 0.02 & 0.03 & 0.03 \\
\hline & Min & 3.02 & 0.53 & 1.2 & 0.67 & 0.17 & 0.86 & 0.52 & 0.3 & 1.12 & 1.79 & 0.4 & 0.77 & 0.37 & 1.35 & 1.61 \\
\hline & $\operatorname{Max}$ & 3.77 & 0.61 & 1.41 & 0.73 & 0.27 & 0.99 & 0.58 & 0.37 & 1.28 & 1.94 & 0.54 & 0.79 & 0.41 & 1.44 & 1.7 \\
\hline \multirow[t]{4}{*}{$\mathrm{F}(\mathrm{N}=6)$} & Mean & 3.38 & 0.57 & 1.43 & 0.74 & 0.19 & 0.99 & 0.59 & 0.34 & 1.26 & 1.91 & 0.52 & 0.76 & 0.4 & 1.41 & 1.71 \\
\hline & SD & 0.27 & 0.02 & 0.01 & 0.04 & 0.02 & 0.34 & 0.03 & 0.02 & 0.05 & 0.06 & 0.02 & 0.02 & 0.01 & 0.06 & 0.06 \\
\hline & Min & 2.98 & 0.53 & 1.42 & 0.68 & 0.16 & 0.94 & 0.55 & 0.31 & 1.19 & 1.84 & 0.5 & 0.73 & 0.39 & 1.32 & 1.62 \\
\hline & Max & 3.75 & 0.61 & 1.44 & 0.79 & 0.21 & 1.03 & 0.64 & 0.37 & 1.34 & 2.02 & 0.54 & 0.78 & 0.43 & 1.47 & 1.79 \\
\hline \multicolumn{17}{|l|}{ P. michaili } \\
\hline \multirow[t]{4}{*}{$\mathrm{M}(\mathrm{N}=2)$} & Mean & 4.05 & 0.54 & 1.29 & 0.76 & 0.21 & 1.02 & & & 1.33 & 2.03 & 0.48 & 0.83 & 0.34 & 1.5 & 1.84 \\
\hline & SD & 0.08 & 0.03 & 0.01 & 0.01 & 0.02 & 0.07 & & & 0.04 & 0.02 & 0.0 & 0.01 & 0.02 & 0.02 & 0.06 \\
\hline & Min & 0.82 & 0.52 & 1.29 & 0.75 & 0.2 & 0.97 & & & 1.31 & 2.01 & & 0.82 & 0.32 & 1.48 & 1.79 \\
\hline & Max & 0.84 & 0.56 & 1.3 & 0.77 & 0.22 & 1.07 & & & 1.36 & 2.04 & 0.08 & 0.84 & 0.35 & 1.51 & 1.88 \\
\hline \multirow[t]{4}{*}{$\mathrm{F}(\mathrm{N}=2)$} & Mean & 3.7 & 0.55 & 1.33 & 0.76 & 0.21 & 0.98 & 0.45 & 0.28 & 1.34 & 2.02 & 0.45 & 0.86 & 0.41 & 1.53 & 1.84 \\
\hline & SD & 0.14 & 0.01 & 0.04 & 0.01 & 0.01 & 0.08 & 0.03 & 0.01 & 0.03 & 0.1 & 0.07 & 0.01 & 0.01 & 0.04 & 0.02 \\
\hline & Min & 3.6 & 0.54 & 1.3 & & & 0.92 & & & & 1.96 & & 0.85 & 0.41 & & 1.82 \\
\hline & Max & 3.8 & 0.55 & 1.35 & 0.77 & 0.22 & 1.04 & 0.47 & 0.29 & 1.37 & 2.09 & 0.5 & 0.87 & 0.42 & 1.55 & 1.85 \\
\hline P. roseog & tatus & & & & & & & & & & & & & & & \\
\hline $\mathrm{F}(\mathrm{N}=2)$ & Mean & 3.98 & 0.62 & 1.53 & 0.67 & 0.24 & 1.22 & 0.69 & 0.53 & 1.43 & 2.21 & & 0.78 & 0.41 & 1.41 & 1.82 \\
\hline & SD & 0.01 & 0.02 & 0.04 & 0.08 & 0 & 0.16 & 0.03 & 0.15 & 0.06 & 0.01 & & 0.03 & 0.02 & 0.11 & 0.13 \\
\hline & Min & 3.98 & 0.61 & 1.5 & 0.61 & 0.23 & 1.11 & 0.67 & 0.42 & 1.39 & 2.2 & & 0.76 & 0.4 & 1.32 & 1.73 \\
\hline & Max & 3.99 & 0.64 & 1.56 & 0.73 & 0.24 & 1.33 & 0.71 & 0.63 & 1.47 & 2.21 & & 0.8 & 0.43 & 1.49 & 1.91 \\
\hline P. suwonan & $u s \mathrm{sp} . \mathrm{n}$. & & & & & & & & & & & & & & & \\
\hline $\mathrm{M}(\mathrm{N}=6)$ & Mean & 3.57 & 0.49 & 1.14 & 0.68 & 0.2 & 0.94 & 0.54 & 0.34 & 1.25 & 1.79 & 0.55 & 0.83 & 0.36 & 1.36 & 1.61 \\
\hline & SD & 0.25 & 0.05 & 0.0 & 0.02 & 0.0 & 0.05 & 0.04 & & 0.05 & 0.05 & & 0.02 & 0.004 & 0.02 & 0.05 \\
\hline & Min & 0.35 & 0.41 & 1.1 & 0.64 & 0.18 & 0.88 & 0.51 & 0.32 & 1.21 & 1.71 & 0.5 & 0.81 & 0.36 & 1.34 & 1.53 \\
\hline $\mathrm{F}(\mathrm{N}=6)$ & Mean & 3.68 & 0.57 & 1.15 & 0.71 & 0.2 & 0.96 & 0.53 & 0.34 & 1.31 & 1.88 & 0.54 & 0.84 & 0.39 & 1.41 & 1.75 \\
\hline & $\mathrm{SD}$ & 0.23 & 0.04 & 0.02 & 0.03 & 0.01 & 0.02 & 0.02 & 0.03 & 0.03 & 0.05 & 0.03 & 0.01 & 0.01 & 0.03 & 0.07 \\
\hline & Min & 3.35 & 0.51 & 1.13 & 0.67 & & 0.93 & & & 1.28 & 1.8 & & 0.82 & 0.37 & 1.37 & 1.68 \\
\hline & Max & 4.02 & 0.62 & 1.2 & & 0.22 & 0.99 & 0.55 & 0.38 & 1.34 & 1.94 & 0.57 & 0.86 & 0.41 & 1.45 & 1.87 \\
\hline & nicus & & & & & & & & & & & & & & & \\
\hline $\mathrm{F}(\mathrm{N}=1)$ & Mean & 4.15 & 0.59 & 1.64 & 0.63 & 0.2 & 1.12 & 0.61 & & 1.33 & 2.02 & 0.49 & 0.79 & 0.4 & 1.29 & 1.68 \\
\hline P. tonnicha & nus & & & & & & & & & & & & & & & \\
\hline $\mathrm{M}(\mathrm{N}=5)$ & Mean & 3.1 & 0.53 & 1.21 & 0.61 & 0.21 & 0.94 & 0.53 & 0.36 & 1.13 & 1.78 & 0.49 & 0.74 & 0.36 & 1.21 & 1.51 \\
\hline & $\mathrm{SD}$ & 0.3 & 0.03 & 0.06 & 0.04 & 0.01 & 0.06 & 0.03 & 0.02 & 0.06 & 0.09 & 0.0 & 0.04 & 0.02 & 0.08 & 0.15 \\
\hline & Min & 2.7 & 0.48 & 1.15 & 0.57 & 0.19 & 0.88 & 0.48 & 0.34 & 1.08 & 1.68 & 0.46 & 0.69 & 0.33 & 1.1 & 1.32 \\
\hline & Max & 3.44 & 0.55 & 1.27 & 0.65 & 0.23 & 1.03 & 0.58 & 0.38 & 1.23 & 1.9 & 0.53 & 0.79 & 0.38 & 1.3 & 1.68 \\
\hline $\mathrm{F}(\mathrm{N}=5)$ & Mean & 3 & 0.52 & 1.25 & 0.59 & 0.19 & 0.92 & 0.49 & 0.31 & 1.14 & 1.71 & 0.51 & 0.75 & 0.38 & 1.18 & 1.44 \\
\hline & SD & 0.18 & 0.08 & 0.02 & 0.04 & 0.01 & 0.05 & 0.04 & 0.02 & 0.07 & 0.05 & 0.04 & 0.02 & 0.02 & 0.07 & 0.06 \\
\hline & Min & 2.69 & 0.41 & 1.23 & 0.52 & 0.18 & 0.87 & 0.44 & 0.3 & 1.04 & 1.63 & 0.47 & 0.71 & 0.36 & 1.06 & 1.33 \\
\hline & Max & 3.14 & 0.59 & 1.26 & 0.63 & 0.2 & 1.01 & 0.53 & 0.33 & 1.2 & 1.74 & 0.56 & 0.76 & 0.41 & 1.23 & 1.49 \\
\hline
\end{tabular}

Diagnosis. Entirely black head and pronotum, castaneous black (or black) mesoscutum, scutellum and hemelytra, and pale femora, tibiae with large castaneous red spots (Fig. 1A-B). For a detail description see Josifov, 1983: 198 .

Male genitalia (Figs 2D-H): Endosoma C-shaped, with elongated secondary processes laterally, simple apex with short and slender apical process (Figs 2D-E). Phallotheca narrow sub-apically notched with triangular apex (Fig. $2 \mathrm{~F})$. Left paramere illustrated as in Fig. $2 \mathrm{H}$ and right paramere as in Fig. 2G.

Female genitalia (Figs 13A, 17A): Bursa copulatrix large, dorsal labiate plate with series of spinules laterally and numerous membranous folds anteriorly, and sclerotized rings elongated, and tapered anteriorly (Fig. 13A). Vestibular sclerites as in Fig. 17A.

Measurements. See Table 1.

Specimens examined. Paratypes: North Korea: $2 \hat{0}$, Bagjon, $20 \mathrm{~km}$ N Kaesong, 21.-23.v.1975, M. Josifov, determined by M. Josifov (TLMF). South Korea: Gangwon-do: $3 \sigma^{\star}$, Donghae-si, Mt. Duta, 16.v.2001, light trap (NAAS); 1 웅 Hoengseong-gun, 27.iv.2010, Quercus sp. (Fagaceae), S.H. Lee (nymph emerge) (SNU). Gyeonggi-do: 10, Anyang-si, Gwanak Arboretum, 6.v.2009, R.K. Duwal (SNU); 20 , Paju-si, Musan-eup, Gunnaemyeon, 21.v.2008, on Acer ginnala Maxim. (Aceraceae), S. Jung (SNU); 20 , Gwangju-si, Mt. Taehwa, 2.-20.v.2008, Mal- 


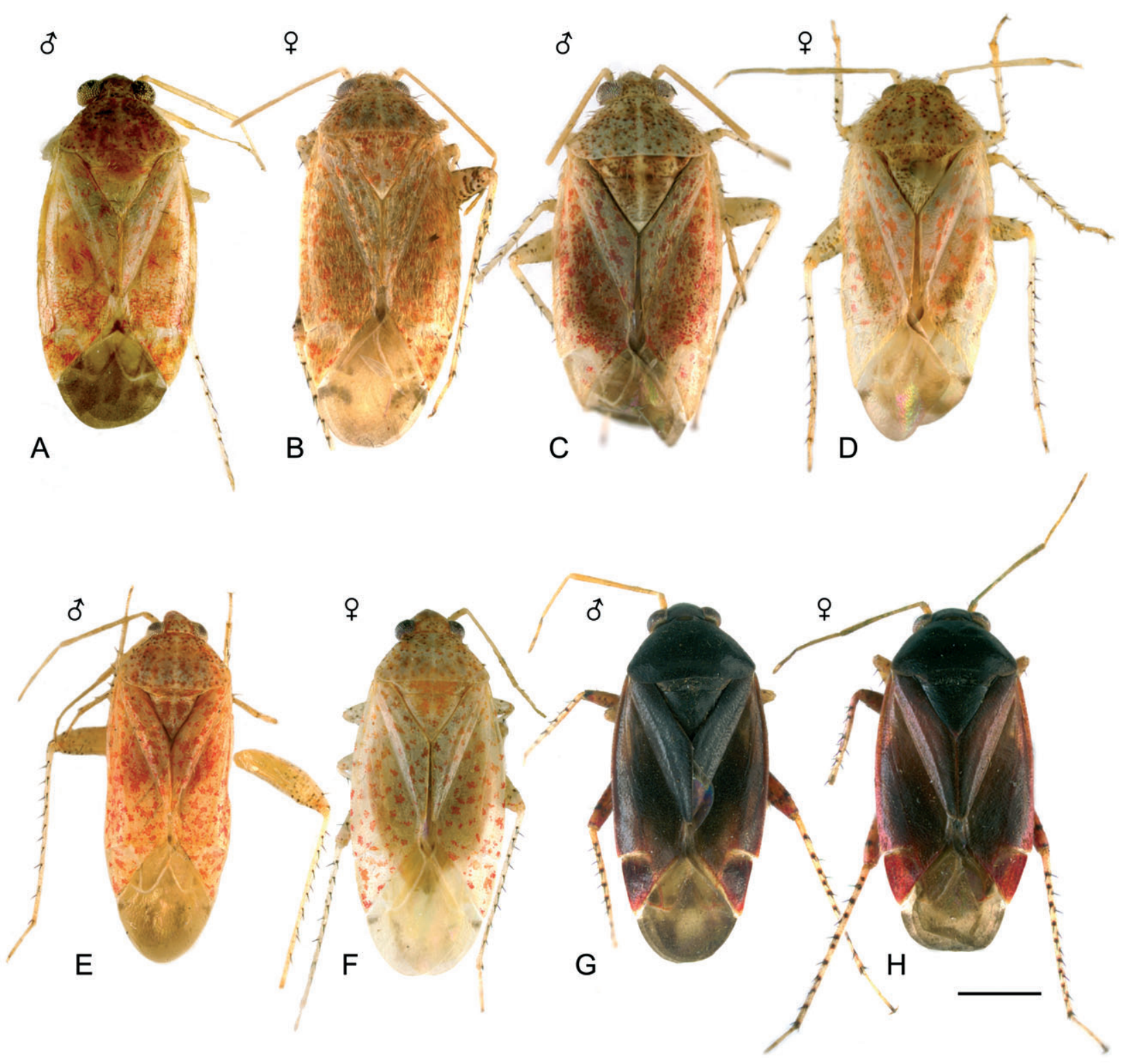

Fig. 4. Habitus images of Psallus spp. A-B - P. roseoguttatus; $\mathrm{C}-\mathrm{D}-$ P. clarus; E-F - P. tesongsanicus; G-H - P. suwonanus sp. n. Scale bar: $0.5 \mathrm{~mm}$.

aise trap, J.O. Lim (SNU); 2ð, Yangpyeong-gun, Mt. Yongmun, 24.vi.2009, light trap, R.K. Duwal and S. Jung (AMNH PBI 00383553) (SNU); $90 \hat{0}, 4 \%$, same collection data as above except for date, 24.vi.2010 (SNU); Jeollanam-do: $5 \hat{0}$, 1 ㅇ, Jangseong-gun, Mt. Bangjang, 24.vi.2010, light trap, R.K. Duwal (AMNH_PBI 00383548-383550; 383551-383552) (SNU).

Distribution. China (Southwest), Korea (North, South).

Biology. Josifov (1983) recorded the host plant as Crataegus sp., Prunus sp. (Rosaceae), whereas in Korea it was collected on a branch of Quercus, then reared in the laboratory during the late winter of 2010. The emerged nymph fed an animal diet, either lepidopteran or coleopteran larvae. It was very active, grew rapidly, and within a week an adult emerged and lived for more than a week. The adult was also observed feeding on decayed plant and animal matter.

\section{Psallus (Apocremnus) atratus Josifov, 1983}

Figs 1C, 2I-M

Psallus (Apocremnus) atratus Josifov, 1983: 197 (desc., figs); 1992a: 116 (list); Kerzhner, 1988a: 845 (key, fig.); Schuh, 1995: 401 (cat.); Kerzhner \& Josifov, 1999: 401 (cat.); Anufriev et al., 2001: 124 (Engl. transl.); Kwon et al., 2001: 178 (cat.).

Diagnosis. Entirely black body, black antennal segment I and distal $(1 / 2$ or $1 / 3)$ of segment II (Fig. 1C), dark ostiolar peritreme, black and shiny femora, and pale tibia with large castaneous black spots. For a detail description see Josifov, 1983.

Male genitalia (Figs 2I-M): Endosoma C-shaped, with broad apex and short angulated apical process, a membranous structure arises from the apex of secondary gonopore that densely covered laterally with spinules, which scattered towards the middle (Figs 2I-J). Phallotheca with wide base, narrow towards the apex (Fig. 2L). Left para- 


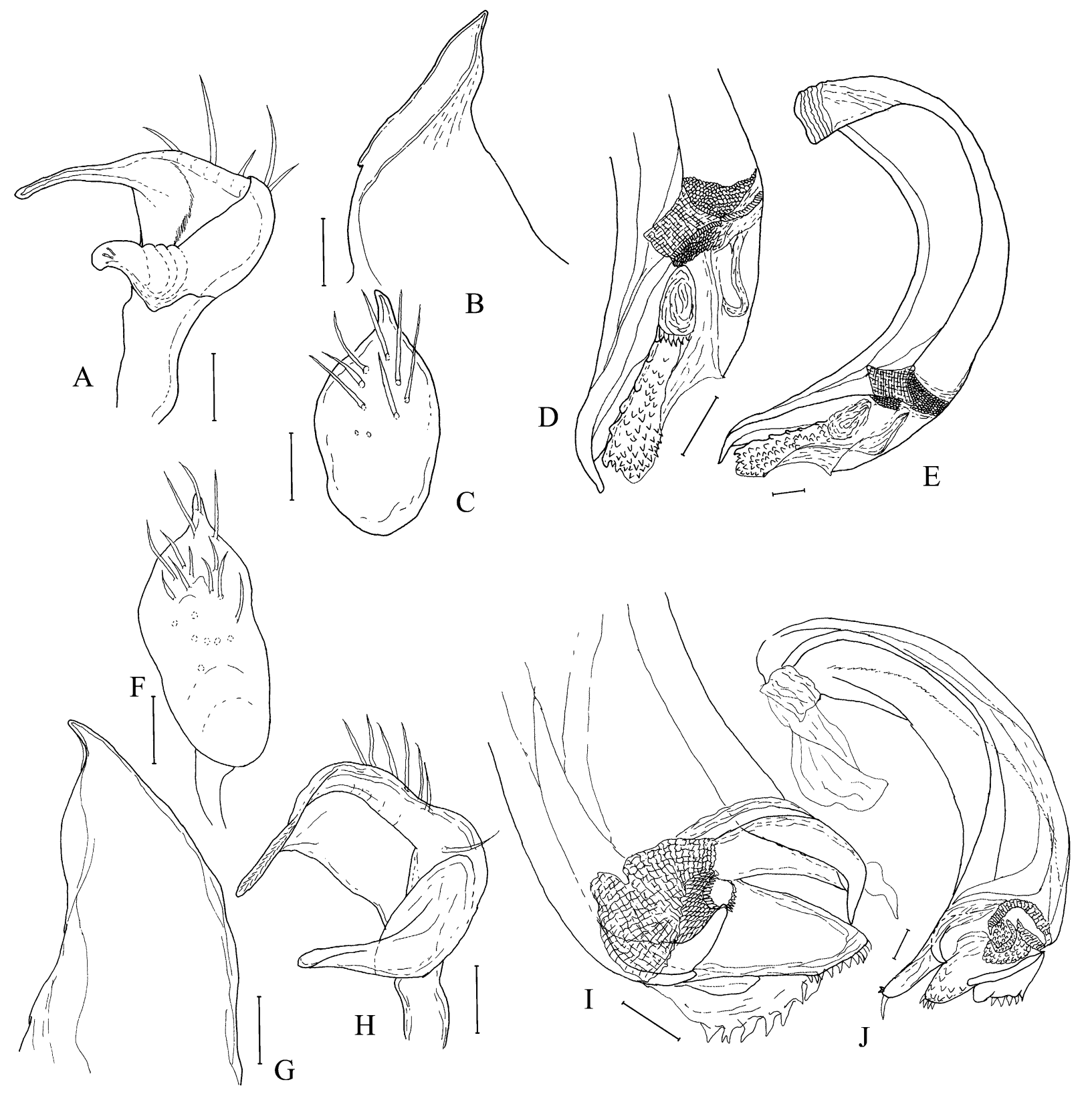

Fig. 5. Male genitalia of Psallus spp. A-E - P. clarus; F-J - P. tesongsanicus. A, H - left paramere; C, F - right paramere; D-E, I-J - endosoma; B, G - phallotheca. Scale bars: $0.1 \mathrm{~mm}$.

mere with a more or less triangular anterior process and an elongate posterior process (Fig. 2M). Right paramere as in Fig. 2K.

Measurements. See Table 1.

Specimens examined. South Korea: Gyeonggi-do: $10,1 \%$, branch with eggs collected from Yangpyeong-gun, 20.iv.2009, Prunus sp. (Rosaceae), R.K. Duwal (AMNH_PBI 00383496; 383495) (SNU) [B in alcohol].

Distribution. Korea (North, South*), Russia (Far East).

Biology. This species is recorded by Josifov (1983) on Crataegus sp. (Rosaceae). Likewise two individuals in this study were observed on a branch of Prunus sp. (Rosaceae) in Korea. The branch was collected during early spring when the temperature was still low. The nymphs that emerged developed into adults that lived for about $10-15$ days.

\section{Psallus (Apocremnus) betuleti (Fallén, 1826)}

Figs 1D, 3A-D

Cimex cruentus Müller, 1776: 108.

Phytocoris betuleti Fallén, 1826: 15.

Psallus betuleti: Zaitzeva, 1968: 866 (desc., fig.); Wheeler \& Henry, 1992: 185 (distr., host); Schwartz \& Kelton, 1990: 946 (diag., desc.); Schuh, 1995: 401 (cat.); Kerzhner \& Josifov, 1999: 401 (cat.); Anufriev et al., 2001 (Engl. transl.); Kwon et al., 2001: 178 (cat.); Rieger \& Rabitsch, 2006: 163 (diag., distr., figs).

Diagnosis. Entirely black body, completely dark antennal segment I and II, black hemelytron tinged with red laterally, castaneous red cuneus with pale base (Fig. 1D), and pale tibiae tinged with red and with small castaneous spots. For a detail description see Schwartz \& Kelton, 1990: 946. 


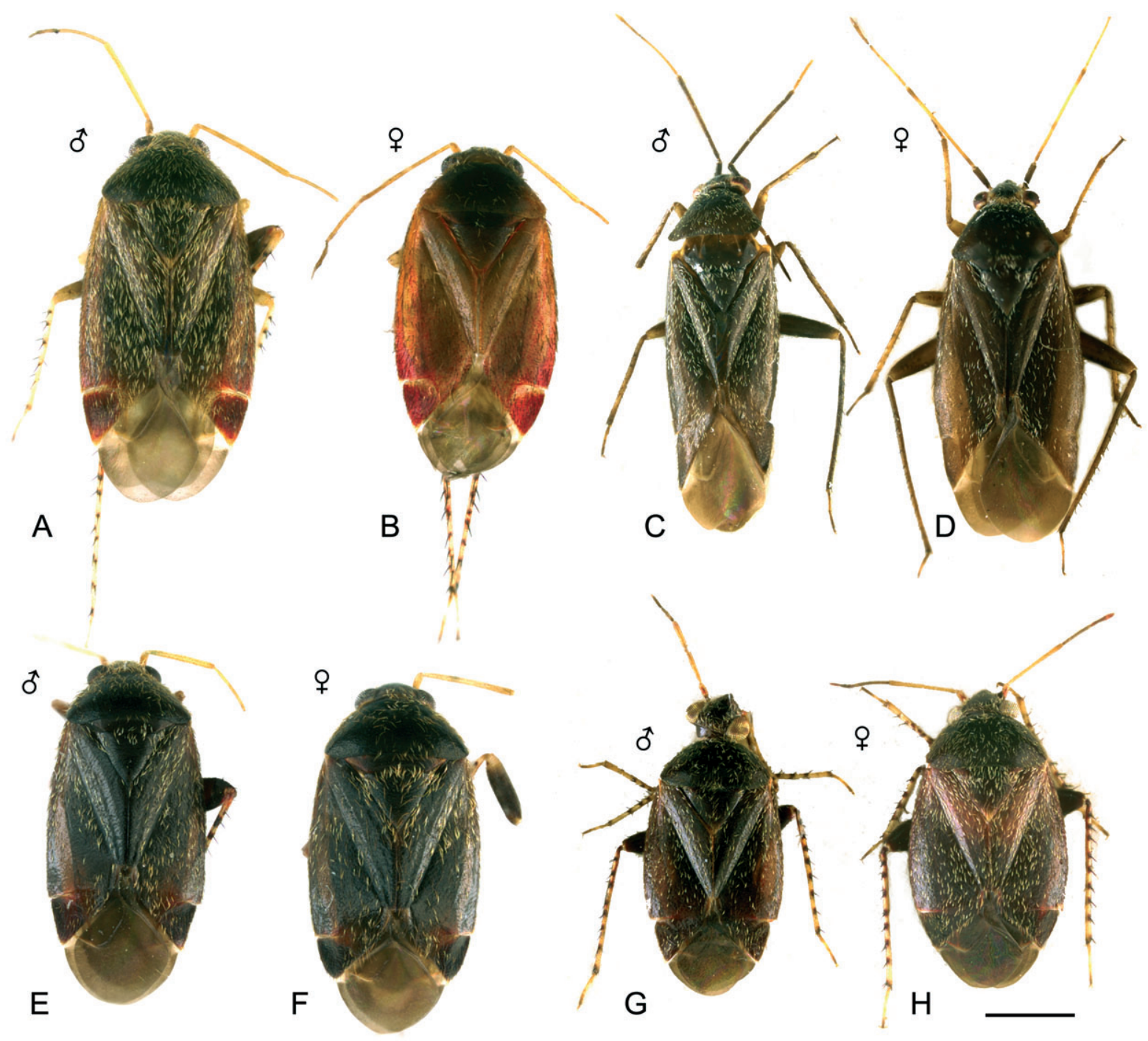

Fig. 6. Habitus images of Psallus spp. A-B - P. tonnaichanus; C-D - P. samdzijonicus; E-F - P. castaneae; G-H - P. ernsti sp. n. Scale bar: $0.5 \mathrm{~mm}$.

Male genitalia (Figs 3A-D): Endosoma more or less S-shaped with elongated apical process and apex of endosoma with a lateral row of spinules (Figs 3C-D). Phallotheca broad, lateral margins nearly parallel (Fig. 3A). Left paramere illustrated as in Fig. 3B.

Specimens examined. Russia: S. Yakutia: $1 \hat{\delta}$, Lengra, Mt. Stanovoj, 17.-18.vii.1995, on Alnus sp. (Betulaceae), T. Yasunaga, determined by T. Yasunaga, 2008 (AMNH_PBI 00383497) (SNU).

Distribution. China (North), Korea (North), Russia (Sakhalin Is., Kurile Is., Siberia), Canada (North), Europe, America (North).

Biology. This species is reported from various host plants; Betula populifolia Marsh (Betulaceae) (Henry \& Wheeler, 1979), Alnus spp. (Betulaceae) (Kerzhner, 1978), Epilobium (Onagraceae) and Rhododendron (Ericaceae) (Schwartz \& Kelton, 1990) and Yasunaga also collected it on Alnus sp. (Betulaceae).

\section{Psallus (Apocremnus) michaili Kerzhner \& Schuh, 1995}

Figs 1E, 3E-I, 14C, 17B, 18F

Psallus Apocremnus niger Josifov, $1992 \mathrm{~b}: 113$ (junior primary homonym of Psallus graminicola $f$. nigra Stichel, 1956); 1992a: 116 (list).

Psallus michaili Kerzhner \& Schuh, 1995: 4 (new name); Schuh, 1995: 409 (cat.); Kerzhner \& Josifov, 1999: 402 (cat.); Anufriev et al., 2001 (Engl. transl.); Kwon et al., 2001: 178 (cat.).

Diagnosis. Completely black body, dorsum and appendages (Fig. 1E), dirty yellow tibiae with large black (or castaneous) spots. For a detail description see Josifov, 1992b.

Male genitalia (Figs 3E-I): Endosoma nearly S-shaped with numerous clusters of spinules and an apical process elongated into a spine on the membranous apex (Figs 3E-F). Phallotheca nearly like a right angle triangle, as in Fig. $3 \mathrm{H}$. Left paramere as in Fig. $3 \mathrm{G}$ and right paramere as in Fig. 3I. 


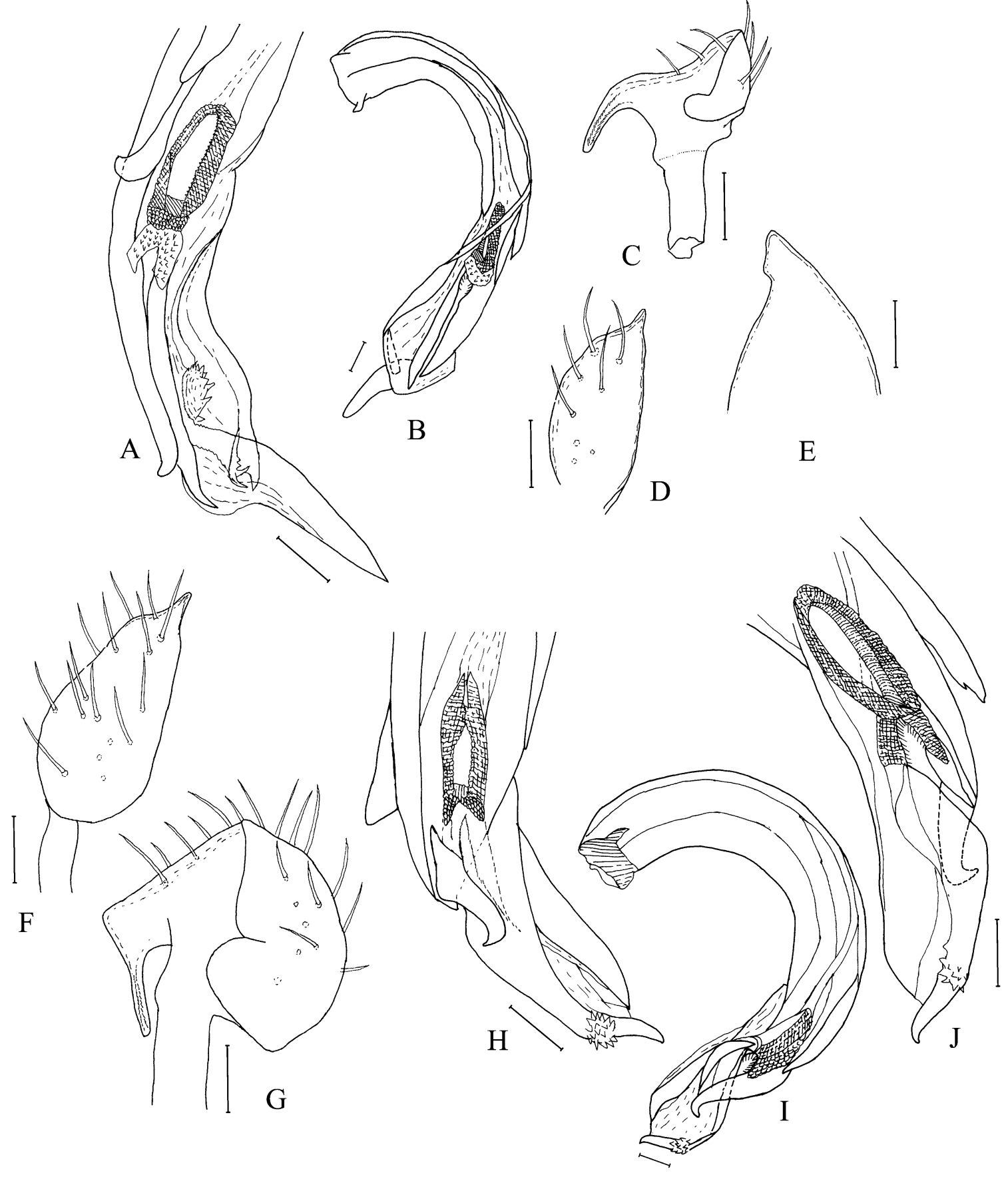

Fig. 7. Male genitalia of Psallus spp. A-E - P. tonnaichanus; F-J - P. suwonanus sp. n. C, G - left paramere; D, F - right paramere; A-B, H-J - endosoma; E - phallotheca. Scale bars: $0.1 \mathrm{~mm}$.

Female genitalia (Figs 14C, 17B, 18F): Bursa copulatrix large, seminal depository wide, dorsal labiate plate with a few spinules laterally, posterior portion of dorsal labiate plate with vase shaped membranous folds, sclerotized rings distinctly elongated and broad (Fig. 14C). Vestibular sclerites illustrated as in Fig. 17B and posterior wall as in Fig. 18F.

Measurements. See Table 1.

Specimens examined. South Korea: Gyeonggi-do: $2 \hat{\sigma}, 2 \%$, Panmunjom, 21.v.2008, Quercus sp. (Fagaceae), S. Jung (AMNH_PBI 00383544-383545; 383546-383547) (SNU).

Distribution. Korea (North, South*).
Biology. Josifov (1992b) documented the host plant of this species as Acer ginnala (Aceraceae) in North Korea, whereas in South Korea it was collected on oak trees (Fagaceae).

\section{Subgenus Calopsallus Yasunaga \& Vinokurov, 2000}

The subgenus Callopsallus was introduced by Yasunaga \& Vinokurov (2000) with the description of a new species, Psallus (Callopsallus) roseoguttatus from Japan, and along with three East Asian species, $P$. clarus Kerzhner, 1988, P. tesongsanicus Josifov, 1983 and $P$. guttatus Zheng \& Li, 1900 are placed in the same sub- 


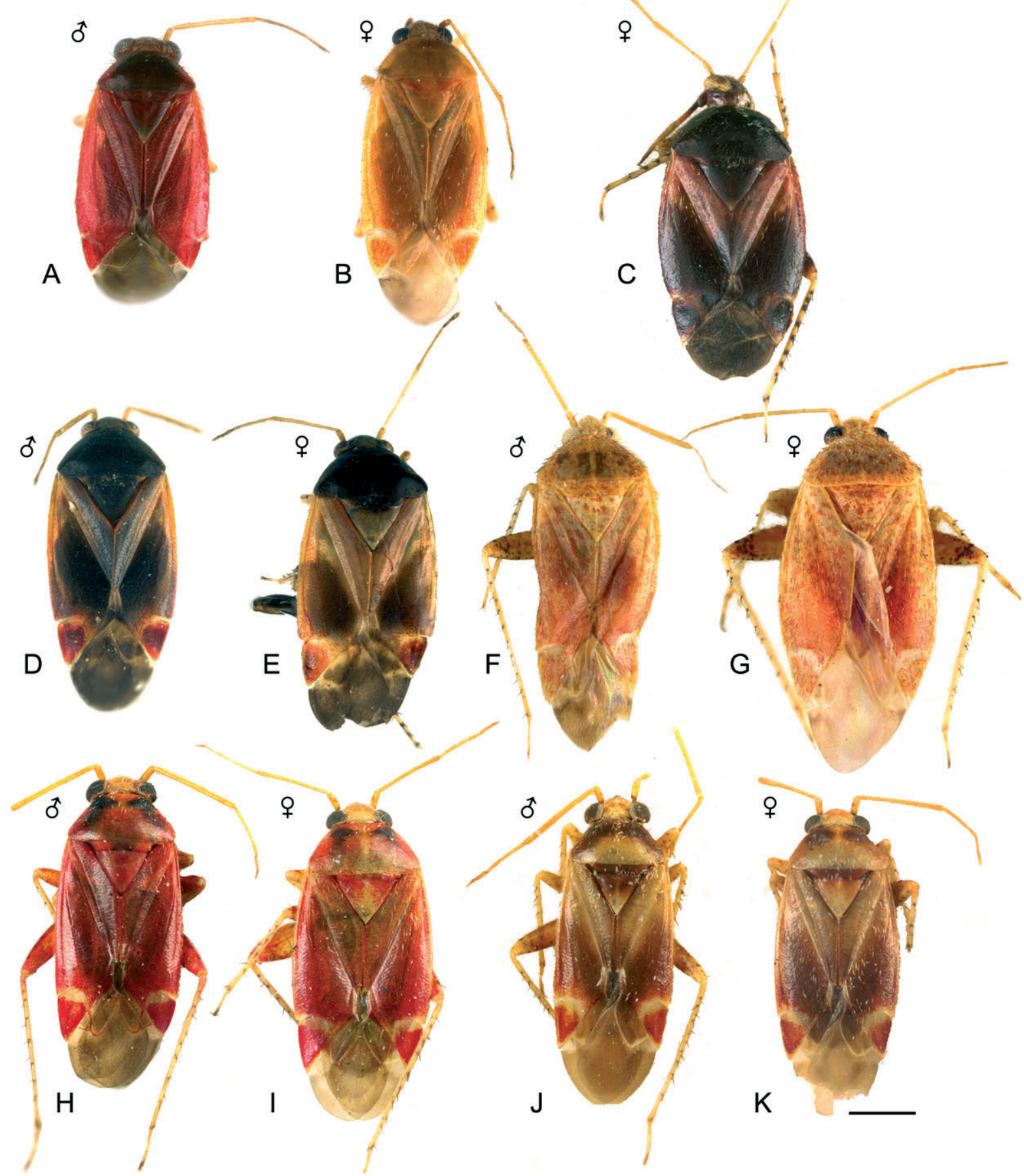

Fig. 8. Habitus images of Psallus spp. A - P. cinnabarinus; B - P. flavescens; C - P. kerzhneri; D-E - P. loginovae; F-G - P. amoenus; $\mathrm{H}-\mathrm{K}-P$. ulmi. Scale bar: $0.5 \mathrm{~mm}$.

genus because of their similar morphology and structure of endosoma.

Members of this subgenus are similar in general appearance to those in the subgenus Psallus. However, they bears specific characteristics, head, pronotum, mesoscutum and scutellum with numerous, small, dark spots; frons with several rows of punctures laterally; pygophore with paired bundles of stiff setae; endosoma broad with very complex apex bearing clusters of spinules; bursa copulatrix with small, oval sclerotized rings, and centrally with semi-sclerotized and membra- nous structures (Fig. 14D-E). For a detail description see Yasunaga \& Vinokurov, 2000.

\section{Psallus (Calopsallus) clarus Kerzhner, 1988}

Figs 4C-D, 5A-E, 14D, 16F

Psallus (Psallus) clarus: Kerzhner, 1988a (March): 845 (key, fig.); 1988b (April): 61 (species description, figs); Schuh, 1995: 403 (cat.); Kerzhner \& Josifov, 1999: 412 (cat.); Anufriev et al., 2001: 123 (Engl. transl.); Kwon et al., 2001: 182 (cat.) 


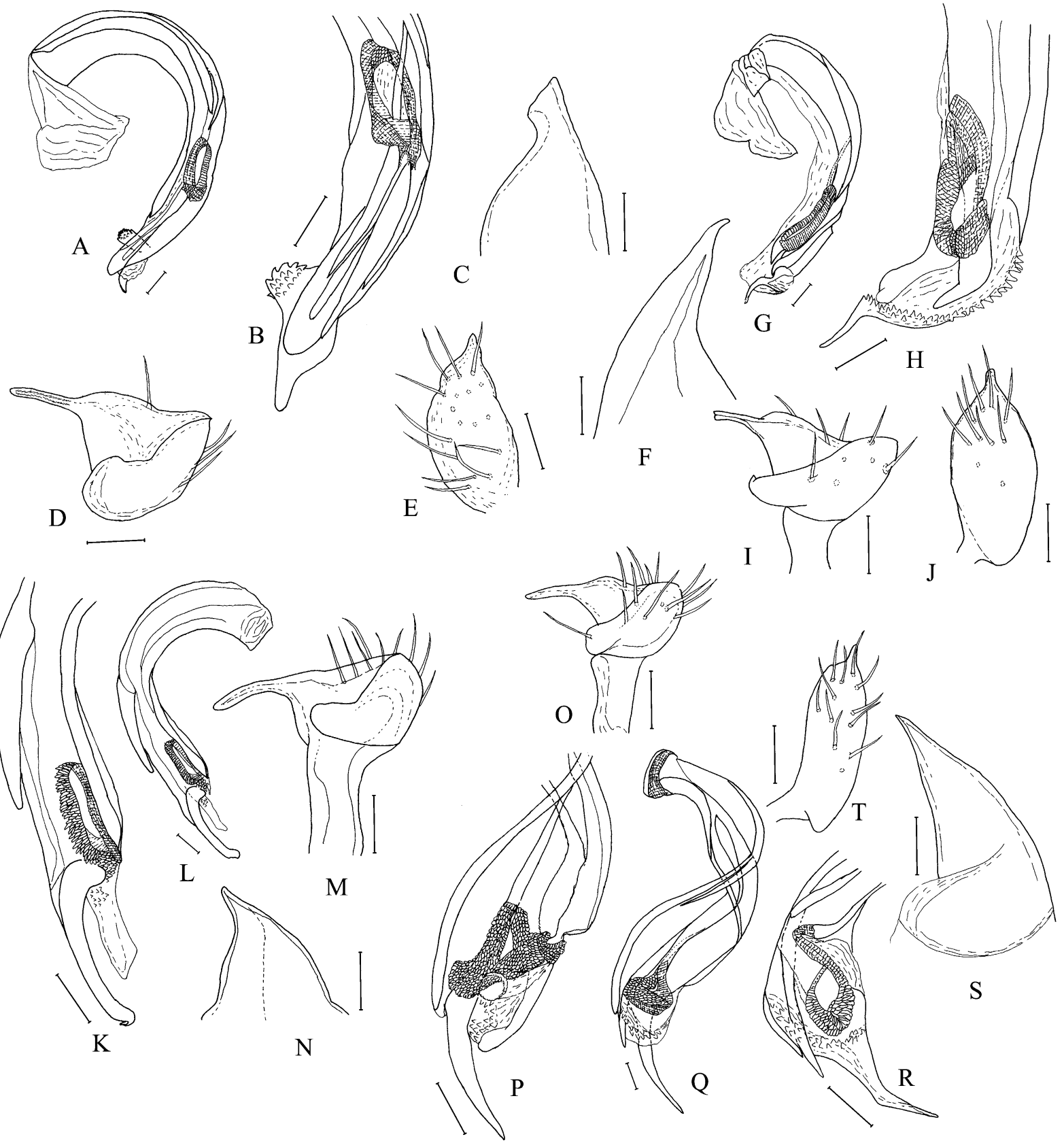

Fig. 9. Male genitalia of Psallus spp. A-E - P. castaneae; $\mathrm{F}-\mathrm{J}-P$. cinnabarinus; $\mathrm{K}-\mathrm{N}-P$. flavescens; $\mathrm{O}-\mathrm{T}-P$. ernsti $\mathrm{sp} . \mathrm{n} . \mathrm{D}, \mathrm{I}$, M, O - left paramere; E, J, T - right paramere; A-B, G-H, K-L, P-R - endosoma; C, F, N, S - phallotheca. Scale bars: $0.1 \mathrm{~mm}$.

Psallus (Callopsallus) clarus: Yasunaga \& Vinokurov, 2000: 662 (disc.).

Diagnosis. Pale body with a few scattered red speckles on head and pronotum, and densely distributed on hemelytron, brown spots scattered on head, pronotum, mesoscutum and scutellum, entirely pale antennae with brown basal ring on segment I, and pale femora with irregularly arranged ventral spots (Fig. 4C-D). For a detail description see Kerzhner, 1988b.

Male genitalia (Figs 5A-E): Endosoma C-shaped, tubular with blunt apex, spinulus membrane arises from the apex of the secondary gonopore (Figs 5D-E). Phallotheca illustrated as in Fig. 5B, left paramere as in Fig. $5 \mathrm{~A}$ and right paramere as in Fig. $5 \mathrm{C}$.
Female genitalia (Figs 14D, 16F): Bursa copulatrix large, lateral oviducts posteriorly supported by bowl shaped membranous fold, dorsal labiate plate with clusters of spinules laterally, sclerotized rings very small, more or less rounded (Fig. 14D). Vestibular sclerites illustrated as in Fig. 16F.

Measurements. See Table 1.

Specimens examined. South Korea: Chungcheonbuk-do: 1 , Cheongju-si, 17.v.1997, I.H. Lee (SNU). Chungcheongnam-do: 20, Cheonan-si, Ipjang-myeon, Heukam-ri, 13.v.2006, S.W. Park (AMNH_PBI 00383507-383508) (SNU). Gyeonggi-do: 1 을 Icheon-si, 21.v.2008, S. Jung (SNU); 1 \% , Namyangju-si, Jinjeop-eup, Palya-ri, 26.v.2006, J.W. Seong (AMNH_PBI 00383510) (SNU); $1 \delta^{\hat{\sigma}}$, Namyangju-si, Onam-eup, Yangji-ri,

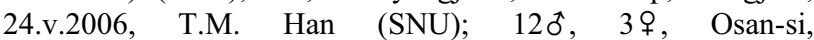



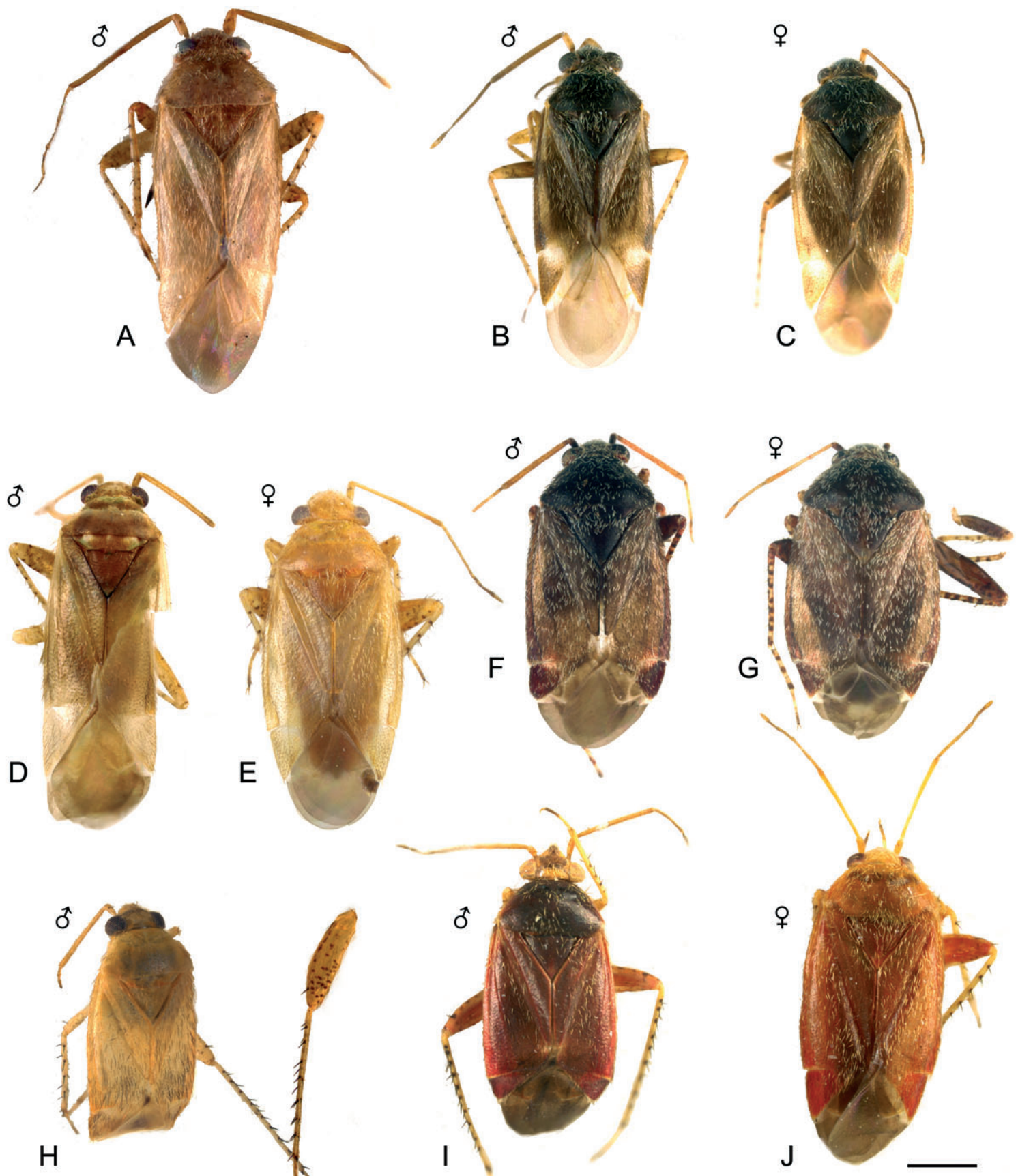

$\mathrm{H}$
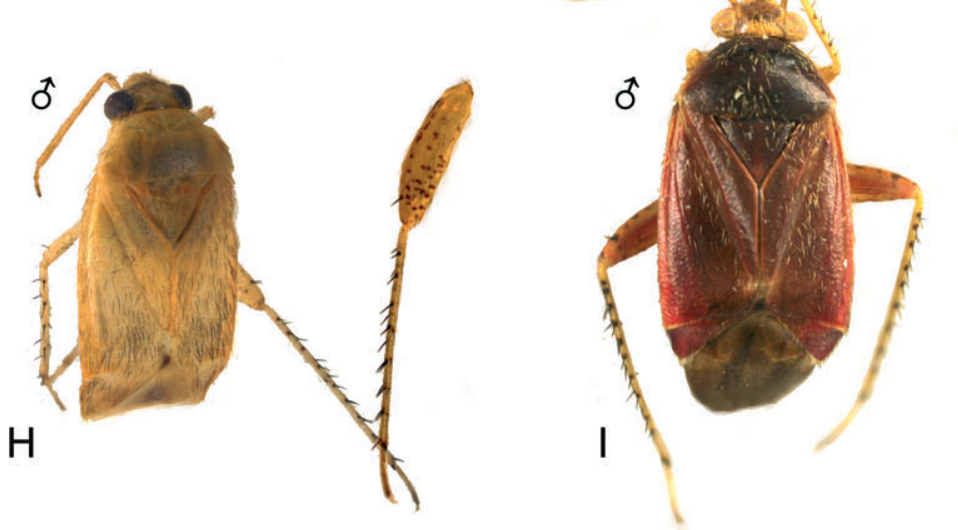

Fig. 10. Habitus images of Psallus spp. A - P. luridus; B-C - P. vittatus; D-E - P. kimi; F-G - P. bagjonicus; $\mathrm{H}-P$. cheongtaensis sp. n.; I-J - P. koreanus. Scale bar: $0.5 \mathrm{~mm}$.

Sucheong-dong, 20.v.1998, light trap, H.K. Lee (AMNH PBI 00383501-383504; 383505-383506, 383514) (SNU); 10, Osan-si, 24.v.2000, light trap, NAAS (SNU); 10, Suwon-si, 10.-11.v.1997, light trap (NAAS); 1 , Seoul, Gwanak-gu, Silim-dong, SNU (CALS), 9.v.2006, light trap, J.W. Seong (AMNH PBI 00383509) (SNU); 10 , Suwon-si, SNU Arboretum, 20.v.2009, on Kalopanax septemlobus (Thunb. ex Murray) (Araliaceae), R.K. Duwal (AMNH PBI 00383511) (SNU); 10 , 1 \%, same data as above, 20.v.2009, on light, R.K. Duwal (AMNH_PBI 00383512; 383513) (SNU); 1ㅇ, Yangpyeong-gun, Mt. Yongmun, 24.vi.2010, light trap, R.K. Duwal and S. Jung (SNU). Jeollanam-do: $57 \%$, Jangseong-gun, Mt. Bangjang, 24.vi.2010, light trap, R.K. Duwal (AMNH PBI 00383515-383522) (SNU).
Distribution. China (Southwest), Korea (North, South*), Russia (Far East).

Biology. Kerzhner (1988) documented the host plant of this species as Quercus dentata (Fagaceae). The host plant is unknown in Korea however, they were observed on Kalopanax septemlobus (Thunb. ex Murray) (Araliaceae) in the Northern region of South Korea.

\section{Psallus (Calopsallus) roseoguttatus Yasunaga \& Vinokurov, 2000}

Figs 3I-L, 4A-B, 14E, 17F, 18B, 19A 

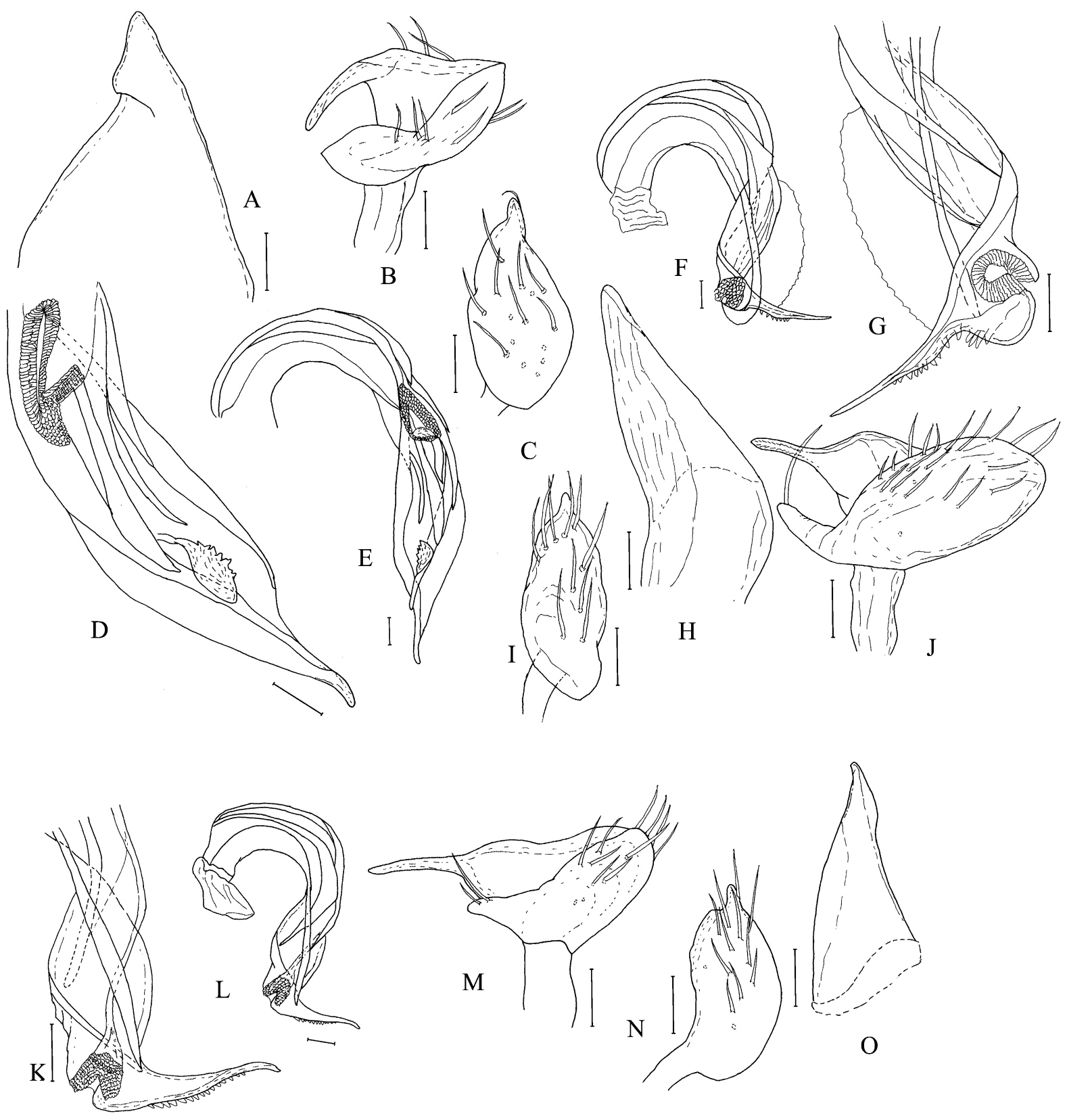

Fig. 11. Male genitalia of Psallus spp. A-E - P. loginovae; F-J - P. luridus; K-O - P. vittatus. B, J, M - left paramere; C, I, Nright paramere; D-E, F-G, K-L - endosoma; A, H, O - phallotheca. Scale bars: $0.1 \mathrm{~mm}$.

Psallus (Calopsallus) roseoguttatus Yasunaga \& Vinokurov, 2000: 662 (desc., figs); Yasunaga, 2001: 172 (diag., fig).

Diagnosis. Entirely pale and shiny dorsum, with red or orange speckles, pale antennae and legs, yellowish femora apically tinged with red and with densely distributed large and small fused spots ventrally and dorsally, and few spots subapically (Fig. 4, 19A-B). For a detail description see Yasunaga \& Vinokurov, 2000.

Male genitalia (Figs 3J-M): Endosoma C-shaped, apex broad and complex furnished with numerous spinules and inner basal margin of apical process with series of toothed spines (Figs $3 \mathrm{~K}-\mathrm{L}$ ). Phallotheca as in Fig. 3J and left paramere as in Fig. 3M.

Female genitalia (Figs 14E, 17F, 18B): Bursa copulatrix large, anterior portion of dorsal labiate plates wide with spinules close to their margins, lateral oviducts positioned roughly within heart shaped membranous fold supported by an additional leaf like membranous baso-lateral fold, sclerotized rings comparatively small, and oval (Fig. 14E). Vestibular sclerites illustrate as in Fig. 17F and posterior wall as in Fig. 18B.

Measurements. See Table 1.

Specimens examined. Paratypes: South Korea*: Gangwon-do: $2 \%$, Donghae-si, Mt. Duta, 16.v.2001, light trap (NAAS). Jeollanam-do: $4 \%$, Jangseong-gun, Mt. Bangjang, 24.vi.2010, light trap, R.K. Duwal (AMNH_PBI 00383485-383488) (SNU). Japan: 20, Shikoku, Kochi Pref., Sameura, 21.v.2000, M. Takai, determined by T. Yasunaga, 2008 (AMNH_PBI 00383484) (SNU).

Distribution. Japan (Shikoku, Honshu), Korea (South)*. 

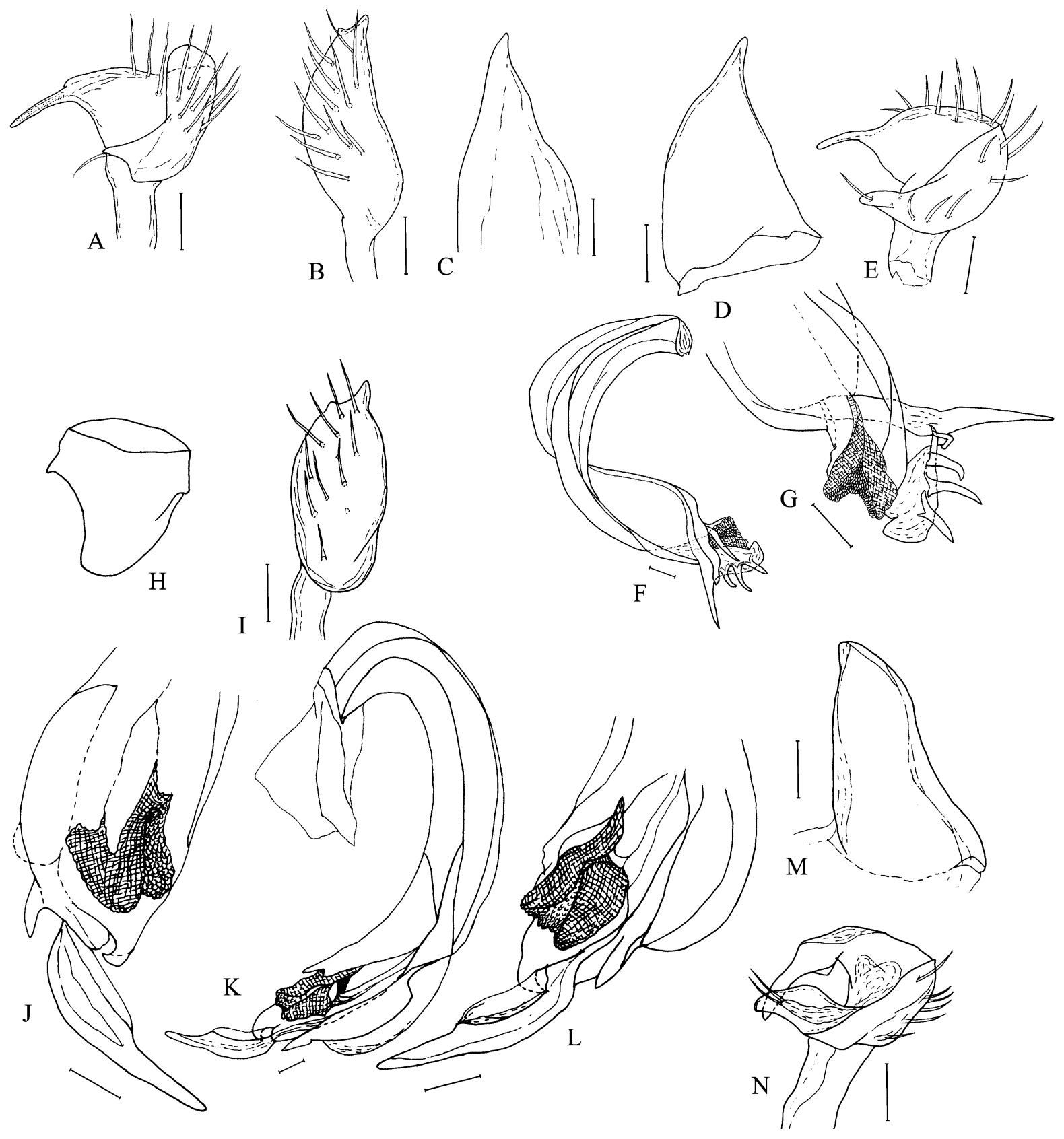

Fig. 12. Male genitalia of Psallus spp. A-C - P. amoenus; D-G - P. bagjonicus; $\mathrm{H}-\mathrm{N}-$ P. cheongtaensis sp. $\mathrm{n} . \mathrm{A}, \mathrm{E}, \mathrm{N}-$ left paramere; B, I - right paramere; F-G, J-L - endosoma; C, D, M-phallotheca; H - pygophore. Scale bars: $0.1 \mathrm{~mm}$.

Biology. Psallus roseoguttatus is very similar in general appearance to $P$. clarus, but in careful observation they differ from each other by pattern of brown spots on dorsum and femora.

Yasunaga \& Vinokurov (2000) recorded the host plant as Quercus serrata (Fagaceae), but in Korea hosts are unknown as all specimens were collected at light (NAAS).

\section{Psallus (Calopsallus) tesongsanicus Josifov, 1983}

Figs 4E-F, 5F-J, 15A

Psallus (Psallus) tesongsanicus Josifov, 1983: 207 (desc., figs); 1992a: 116 (list); Schuh, 1995: 416 (cat.); Kerzhner \& Josifov, 1999: 418 (cat.); Kwon et al., 2001: 183 (cat.).
Psallus (Callopsallus) tesongsanicus: Yasunaga \& Vinokurov, 2000: 662 (desc.).

Diagnosis. Pale or yellowish body, uniformly distributed red speckles on dorsum, scattered brown spots on head, anterior region of pronotum and scutellum, pale femora ventrally with numerous small spots on distal half and dorsally with a few spots subapically (Fig. 4E-F). For a detail description see Josifov, 1983.

Male genitalia (Figs 5F-J): Endosoma C-shaped, broad, apex wide and complex with several short outgrowths with minute spinules. Phallotheca as in Fig. 5G. Left paramere illustrated as in Fig. $5 \mathrm{H}$ and right paramere as in Fig. 5F. 

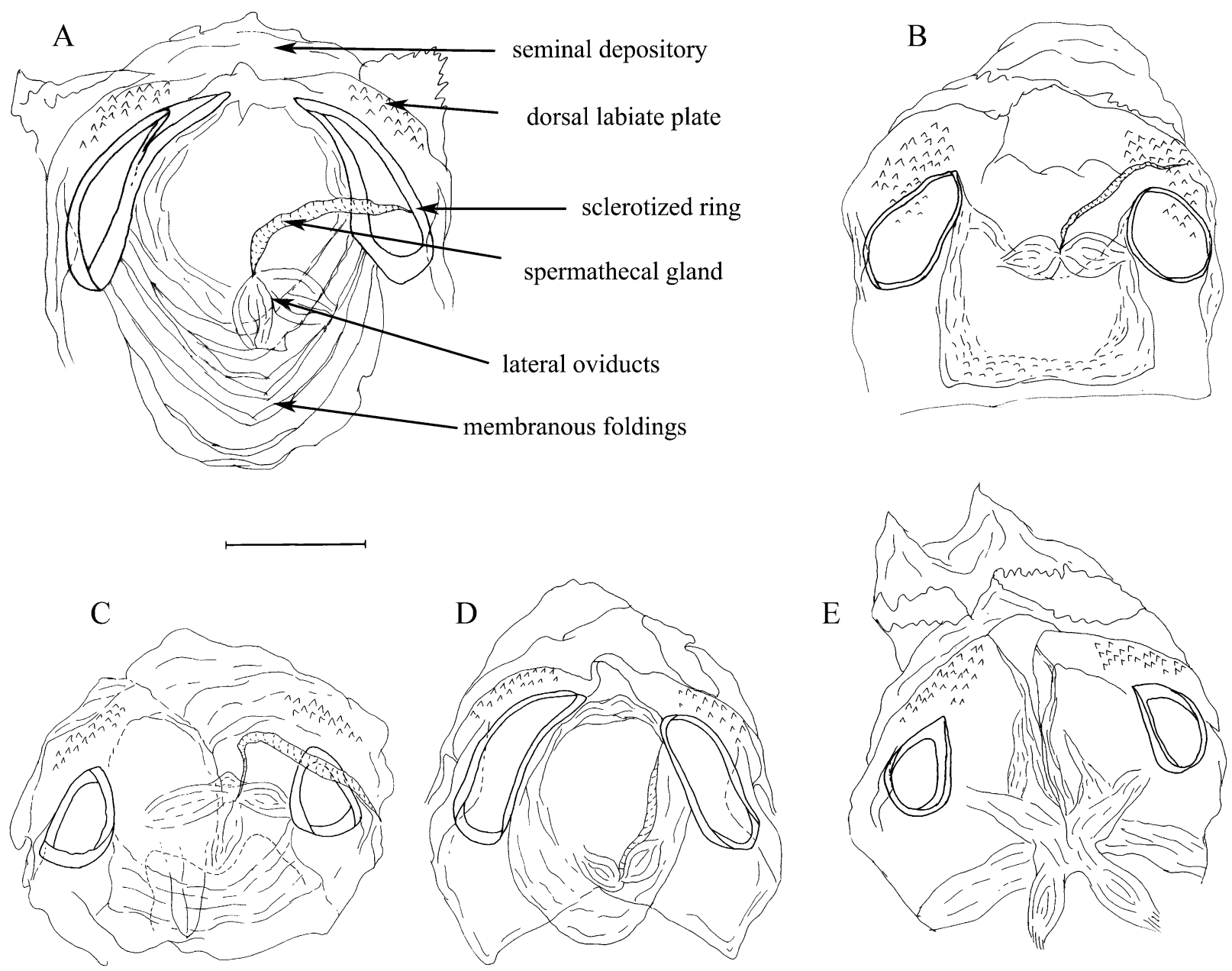

Fig. 13. Female genitalia of Psallus spp. Bursa copulatrix, dorsal view. A - P. ater; B - P. amoenus; C - P. bagjonicus; D - P. castaneae; E - P. cinnabarinus. Scale bar: $0.1 \mathrm{~mm}$.

Female genitalia (Fig. 15A): Bursa copulatrix large, dorsal labiate plate invaginated at middle and with a few spinules laterally, sclerotized rings ovate but posterior margin straight; lateral oviduct supported by membranous fold arising from the dorsal labiate plate.

Measurements. See Table 1.

Specimens examined. North Korea: $2 \hat{\jmath}$, Bei Phiongjang, Rjonggang, Mt. Rjongak, 31.v.1987, M. Josifov, determined by M. Josifov (SNU). South Korea: Gyeonggi-do: 1 + , Yangpyeong-gun, Mt. Yongmun, 11.iv.2009, light trap, R.K. Duwal and S. Jung (AMNH_PBI 00383489) (SNU).

Distribution. Korea (North, South*).

Biology. Josifov (1983) reported this species on Quercus dentata (Fagaceae) in North Korea.

\section{Subgenus Hylopsallus Wagner, 1952}

The subgenus Hyalopsallus is a Holarctic group, which consists of 14 documented species (Kerzhner \& Josifov, 1999; Yasunaga \& Vinokurov, 2000) and one additional species described herein, $P$. suwonanus $\mathrm{sp} . \mathrm{n}$.

Members of this subgenus usually have dark coloured body, pale antennae with dark base on segment I, endosoma C-shaped, short and widened with cluster of spinules at the base of apical process. For a detail description see Wagner, 1952.

\section{Psallus (Hylopsallus) suwonanus sp. n.}

Figs 4G-H, 7F-J, 15C

Holotype. South Korea*: đิ, Gyeonggi-do, Suwon-si, SNU Arboretum, 20.v.2009, on Rhamnus davurica Pallas (Rhamnaceae), R.K. Duwal. Deposited at Biosystematics Laboratory, Seoul National University (AMNH_PBI 00383428) (SNU).

Diagnosis. Blackish or dark brown endocorium, castaneous or deep red exocorium and cuneus, apical $(2 / 3$ or $1 / 2$ ) of femora red with rows of large black spots (Fig. $4 \mathrm{G}-\mathrm{H}$ ), short elongated secondary processes of endosoma furnished with bunch of spinules on subapical region, posteriorly angulated left paramere, and right paramere tapered from base to apex.

Description. Male. Body elongate oval. Coloration (Fig. 4G-H): Head, pronotum, mesoscutum and scutellum black; hemelytron black or blackish red, endocorium black or dark brown, exocorium castaneous or deep red, cuneus castaneous or deep red; membrane brown, with grey apical margin. Ventrally, thoracic region and abdomen black or dark brown except last abdominal seg- 

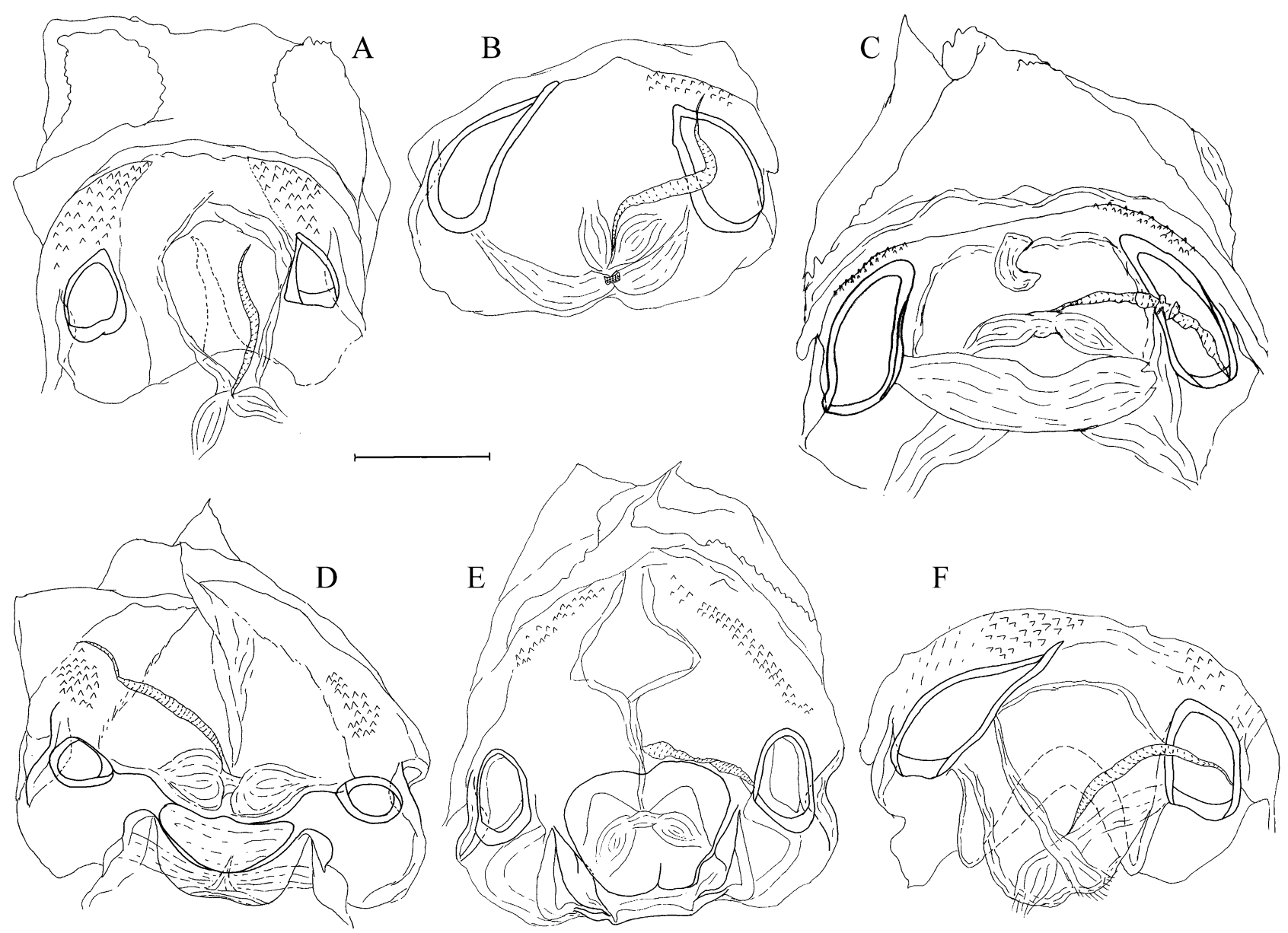

Fig. 14. Female genitalia of Psallus spp. Bursa copulatrix, dorsal view. A - P. flavescens; B - P. kerzhneri; C - P. michaili; D P. clarus; E - P. roseoguttatus; F - P. loginovae. Scale bar: $0.1 \mathrm{~mm}$.

ment castaneous. Antennal segments pale except the base of segment I dark. Labium, segment I, basal half of segment II, apical half of segment IV blackish brown, remaining parts pale. Coxae black, trochanters castaneous; basal $1 / 3$ or $1 / 2$ of metafemora black, apical larger part red with rows of large black spots; tibiae pale with rows of large castaneous spots at the base of black spines. Surface and vestiture: Dorsum furnished with simple black setae and with sericeous setae; head, pronotum, mesoscutum and scutellum slightly shagreened. Structure: Head: Length short compare to body size; interocular space wide; labium slightly surpassing or reaching apex of mesocoxae. Genitalia (Figs 7F-J): Endosoma: shape C-like, with a few long and short secondary processes; a short apical process extending through apex, furnished with a bunch of spinules; secondary gonopore positioned at about 1/4 from the apex. Left paramere: Body short, posterior process more or less angulated, anterior process short and flat. Right paramere: Body elongate, tapered from base to apex, apex with short finger like process.

Female. Not significantly different from male in colour and vestiture. Genitalia (Fig. 15C): Bursa copulatrix of moderate size, dorsal labiate plate with spinules laterally; sclerotized rings large, thin rimmed and somewhat irregular margins; folded membranes arise from anterior and posterior portions of dorsal labiate plate supporting lateral oviducts.

Measurements. See Table 1.

Specimens examined. Paratypes: South Korea: $13 \hat{0}, 30 \%$, same data as for holotype, (AMNH_PBI 00383439-383451; 00383452-383570) (SNU); 19, same data, Pyrus ussuriensis (Rosaceae), R.K. Duwal (SNU).

Etymology. Named after type locality, Suwon.

Distribution. Korea (South)*.

Discussion. Morphologically this species is very similar to Psallus castaneae, or P. tonnaichanus, or $P$. kerzhneri, or $P$. loginovae in colour and vestiture. But it can be separated from $P$. tonnaichanus by the absence of a tuft of stiff setae on each side of the genital segment, and from the other three species by its coloration and spots on the metafemora, which are distinctly different in each species, and the structure of its genitalia (Figs 7A-B, 9A-B, 11D-E).

This species is placed in the subgenus Hylopsallus, based upon the overall appearance of its endosoma being similar to those of congeners, and having a short apical process with the spinulose membrane either at base or middle.

Biology. The confirmed breeding host plant is Rhamnus davurica Pallas (Rhamnaceae); a few individuals were 

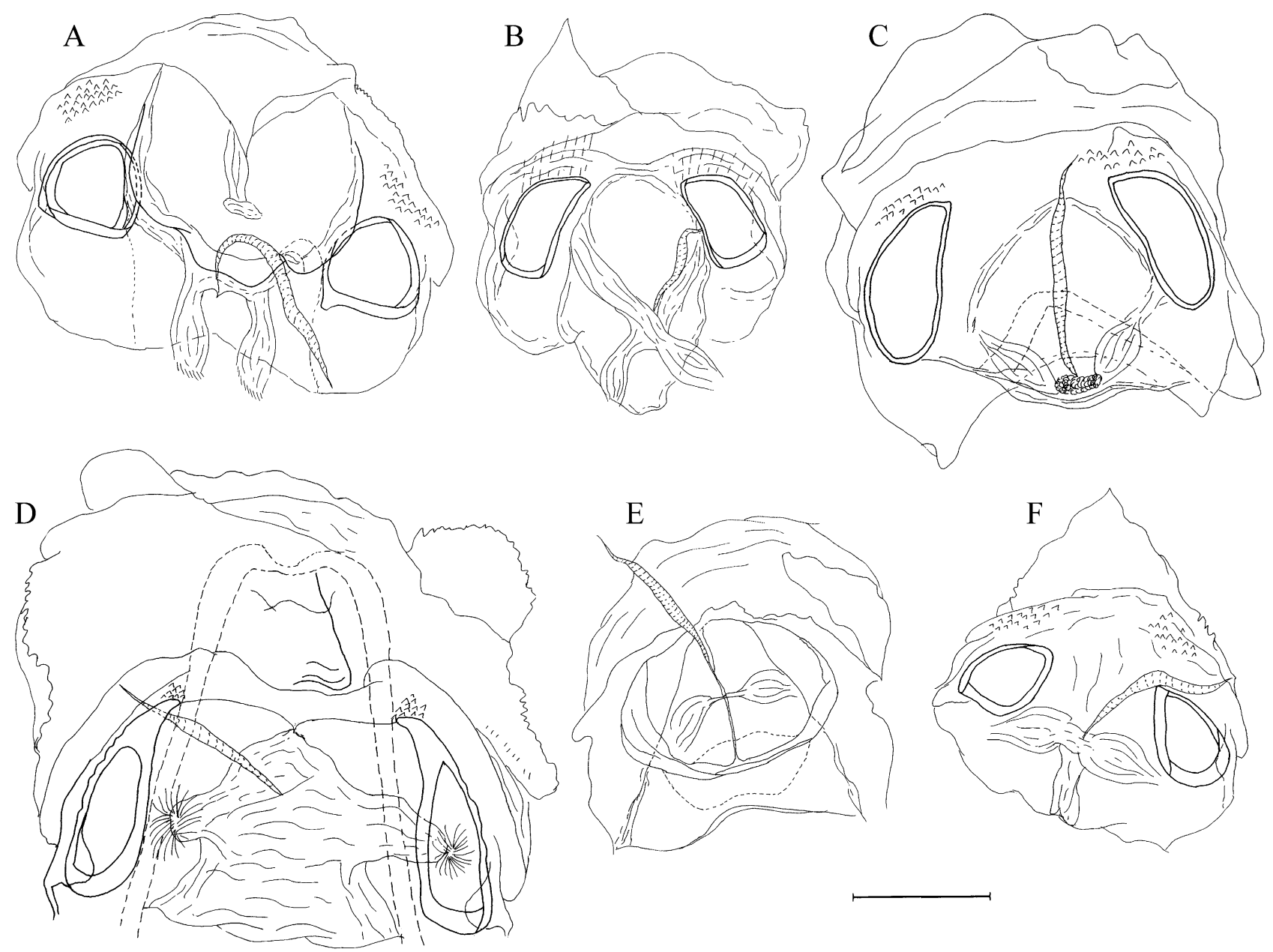

Fig. 15. Female genitalia of Psallus spp. Bursa copulatrix, dorsal view. A $-P$. tesongsanicus; B $-P$. tonnaichanus; $\mathrm{C}-P$. suwonanus sp. n.; D - P. samdzijonicus; E - P. vittatus; F - P. ernsti sp. n. Scale bar: $0.1 \mathrm{~mm}$.

also collected from Pyrus ussuriensis (Rosaceae) which was growing next to the Rhamnus tree. The adults were found to appear from the middle to the end of May. The tree was badly damaged but as this bug was collected together with a large number of plant hoppers, whose secretions made the tree very sticky, it is difficult to assume whether this species is a predator or a pest.

\section{Psallus (Hylopsallus) tonnaichanus Muramoto, 1973}

Figs $6 \mathrm{~A}-\mathrm{B}, 7 \mathrm{~A}-\mathrm{E}, 15 \mathrm{~B}, 16 \mathrm{D}, 18 \mathrm{E}, 19 \mathrm{~B}$

Psallus tonnaichanus Muramoto, 1973: 2897.

Psallus (Phylidea) dryos Kerzhner, 1979: 47 (desc., figs).

Psallus tonnaichanus dolerus Kerzhner, 1988: 74 (comb. n.).

Psallus tonnaichanus dolerus Josifov, $1992 \mathrm{~b}: 117$ (fig.).

Psallus (Hylopsallus) tonnaichanus Yasunaga \& Vinokurov, 2000: 656 (key, distr.); Yasunaga, 2001 (diag., fig.); Anufriev et al., 2001 (Engl. transl.); Kwon et al., 2001: 179 (cat.).

Diagnosis. Reddish dark brown or black dorsum, completely black head and pronotum, entirely pale antennae with extreme base of segment I dark, dark brown hemelytron with red cuneus (Fig. 6A-B), dark brown femora with pale apices and two rows of dark spots arranged ventrally, pale tibiae with large dark brown spots at the base of black spine, and tuft of stiff hairs laterally on pygophore.

Male genitalia (Figs 7A-E): Endosoma C-shaped with an apical process, secondary gonopore apically furnished with minute spinules (Figs 7A-B). Phallotheca illustrated as in Fig. 7E, left paramere as in Fig. 7C and right paramere as in Fig. 7D.

Female genitalia (Figs 15B, 16D, 18E): Bursa copulatrix of moderate size, dorsal labiate plate somewhat sclerotized, membranous fold centrally located between sclerotized rings, sclerotized rings broad and elongate, or somewhat oval elongate. Vestibular sclerites as in Fig. 16D. Posterior wall as in Fig. 18E.

Measurements. See Table 1.

Specimens examined. South Korea: Gangwon-do: 10, Hongcheon-gun, 26.vi.2003, J.W. Seong (AMNH PBI 00383538) (SNU). Gyeonggi-do: 10ે, Yangpyeong-gun, Mt. Yongmun, 11.vi.2009, on light, R.K. Duwal and S. Jung (AMNH_PBI 00383539) (SNU); 2 , same data except for host, Quercus sp. (Fagaceae) (AMNH_PBI 00383540) (SNU); 5 , same data except for date, 24.vi.2009 (AMNH_PBI 00383541, 383543); 1 o, Yongin-si, Mt. Taehwa, 2.-20.vi.2008, Malaise trap, J.O. Lim (SNU). Jeju-do: 40, Jeju-si, Ungto-fall, 12.-15.v.2008, T. Yasunaga, R.K. Duwal and S. Jung (AMNH_PBI 00383536-383537) (SNU). Japan: Hokkaido: 20, 

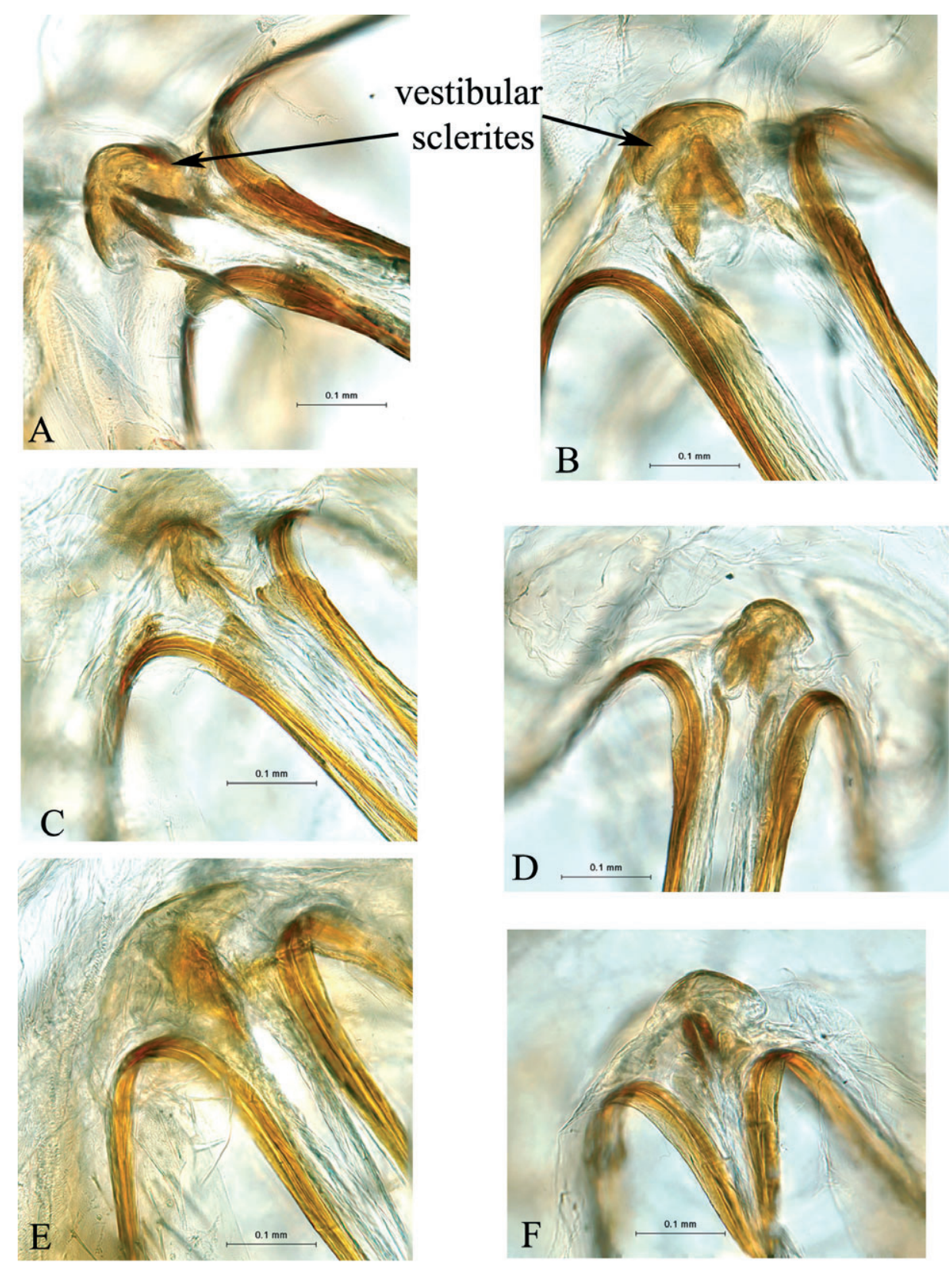

Fig. 16. Female genitalia of Psallus spp. Base of $1^{\text {st }}$ gonapophyses, ventral view. A $-P$. castaneae; B $-P$. loginovae; C $-P$. flavescens; D - P. tonnaichanus; E - P. clarus; F - P. bagjonicus. Scale bars: $0.1 \mathrm{~mm}$.

Mt. Chise-nupuri, 500-600 m alt., Niseko, 9.vii.1994, T. Yasunaga, determined by T. Yasunaga, 2008 (AMNH_PBI 00383542) (SNU).

Distribution. China (Central), Japan (Hokkaido, Honshu, Kyushu), Korea (North, South), Russia (Sakhalin Is., Kurile Is., Kunashir Is.).

Biology. Josifov (1992b), Kerzhner \& Josifov (1999) treat this Korean species as Psallus tonnaichanus dolerus Kerzhner, 1979.

Kerzhner (1988) reported this species on Quercus spp. (Fagaceae), which was confirmed as a breeding host by Yasunaga \& Vinokurov (2000). However, the later author also observed it on flowers of Hydrangea sp. (Hydrangeaceae), Syringa reticulate (Blume) (Oleaceae).

\section{Subgenus Mesopsallus Wagner, 1970}

The subgenus Mesopsallus comprises 4 species (Kerzhner \& Josifov, 1999; Rizzotti, 2000), of which two species, P. samdzijonicus and P. holomelas occur in East Asia.

Members of this genus are comparatively large and elongate, with simple and slender endosoma (different than Psallus type). For a detail description see Wagner, 1970.

\section{Psallus (Mesopsallus) samdzijonicus Josifov, 1983} Figs $6 \mathrm{C}-\mathrm{D}, 15 \mathrm{D}, 17 \mathrm{D}$

Psallus (Mesopsallus) samdzijonicus Josifov, 1983: 200 (desc., figs); 1992a: 116 (list); Schuh, 1995: 415 (cat.); Kerzhner \& 

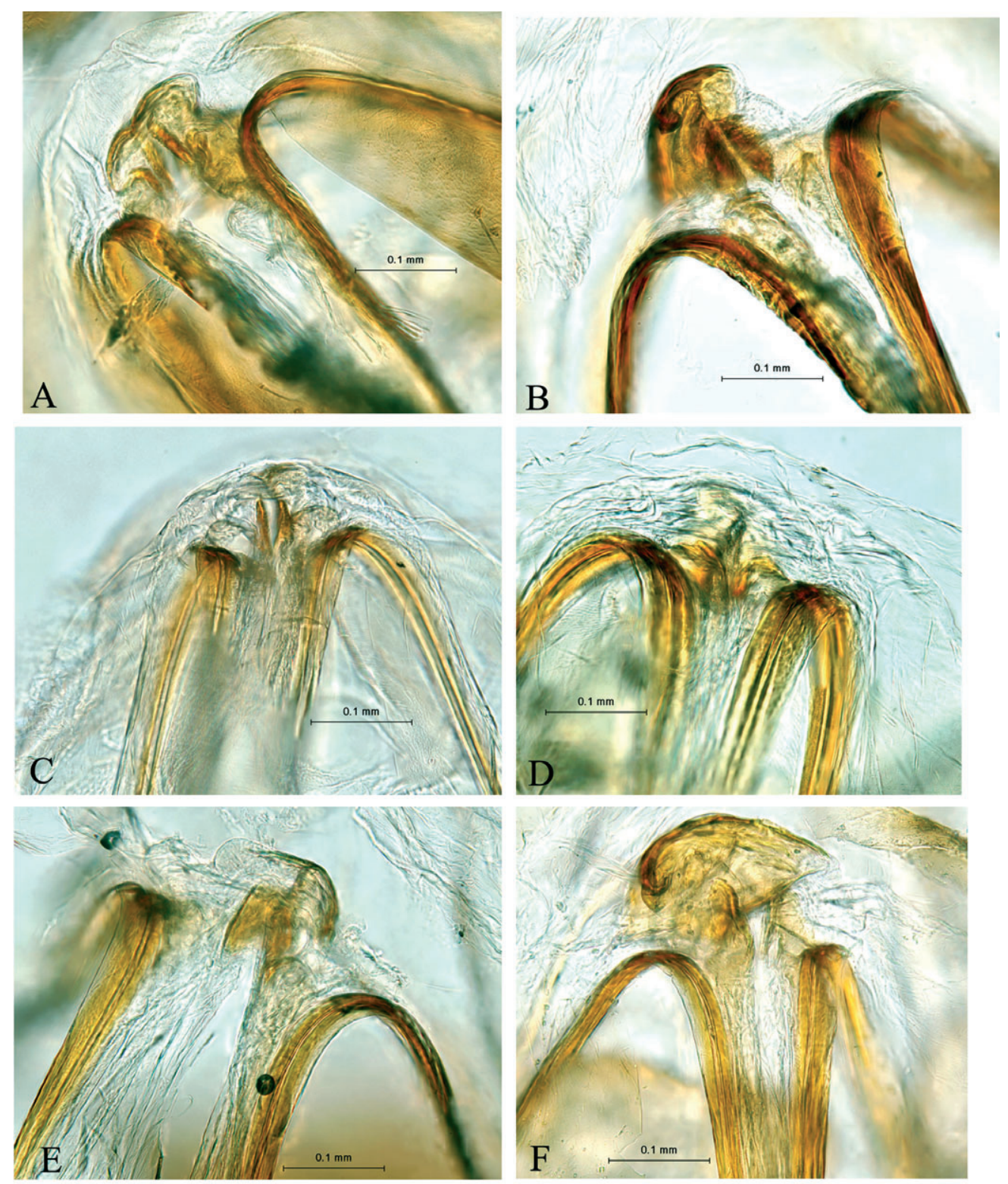

Fig. 17. Female genitalia of Psallus spp. Base of $1^{\text {st }}$ gonapophyses, ventral view. A - P. ater; B - P. michaili; C - P. amoenus; D P. smadzijonicus; E - P. vittatus; F - P. roseoguttatus. Scale bars: $0.1 \mathrm{~mm}$.

Josifov, 1999: 405 (cat.); Anufriev et al., 2001 (Engl. transl.); Kwon et al., 2001: 179 (cat.).

Diagnosis. Large and black body, pale margins of vertex and inner margin of eyes, black antennal segment I, and blackish brown femora with pale apices (Fig. 6C-D). For a detail description and male genitalia see Josifov, 1983: 200.

Female genitalia (Figs 15D, 17D): Bursa copulatrix of moderate size, seminal depository wide, centrally occupied by wide folded membrane, sclerotized rings elongate oval, anterior apex extended into a protuberance that reaches lateral spinules (Fig. 15D). Vestibular sclerites as in Fig. 17D.

Specimens examined. Paratypes: North Korea: $1 \hat{0}, 19$, Jongkang-do, Samdzijon, 13.-19.vii.1974, on Sorbus amurensis Koehne (Rosaceae), M. Josifov, determined by M. Josifov (TLMF).
Distribution. Korea (North).

Biology. Josifov (1983) reported this species on Sorbus amurensis (Rosaceae) in North Korea.

\section{Subgenus Phylidea Reuter, 1899}

Subgenus Phylidea is widely distributed in Holarctic region and comprises of about twenty eight species (Kerzhner \& Josifov, 1999; Yasunaga \& Josifov, 2000).

Members of this subgenus have variously coloured body (bright or dark), smooth or speckled dorsum, broad and short endosoma with series of spinules located subapically or apically. For a detail description see Seidenstücker, 1962.

\section{Psallus (Phylidea) castaneae Josifov, 1983}

Figs 6E-F, 9A-E, 13D, 16A

Psallus (Phylidea) castaneae Josifov, 1983: 202; 1992a: 116 (list); Schuh, 1995: 402 (cat.); Kerzhner \& Josifov, 1999: 405 


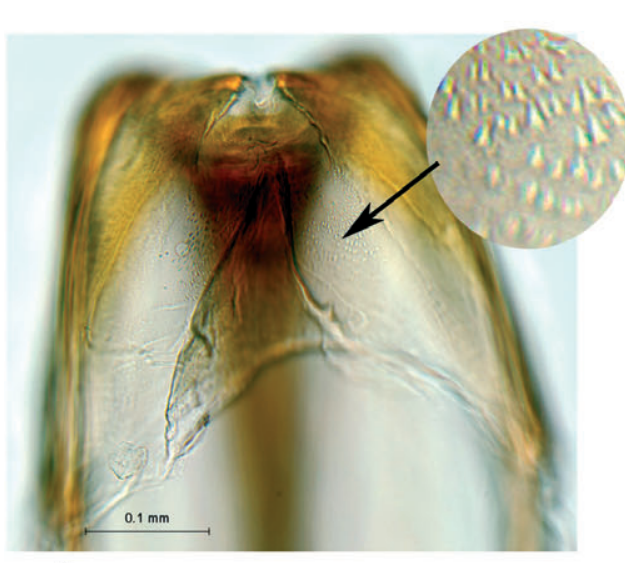

A

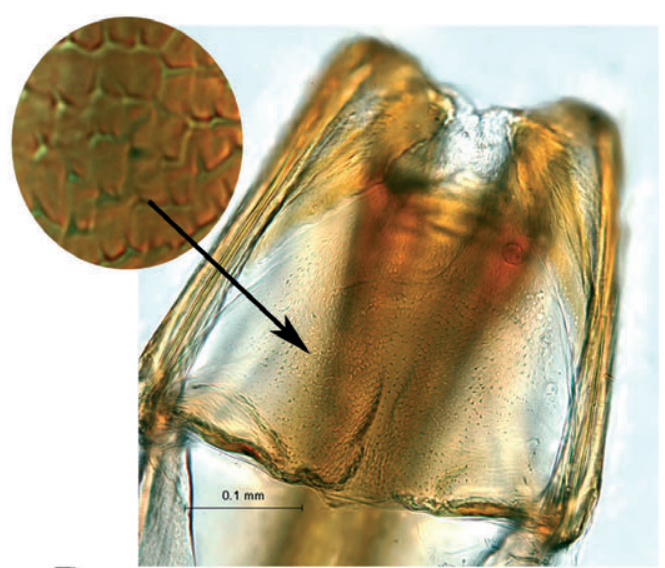

B

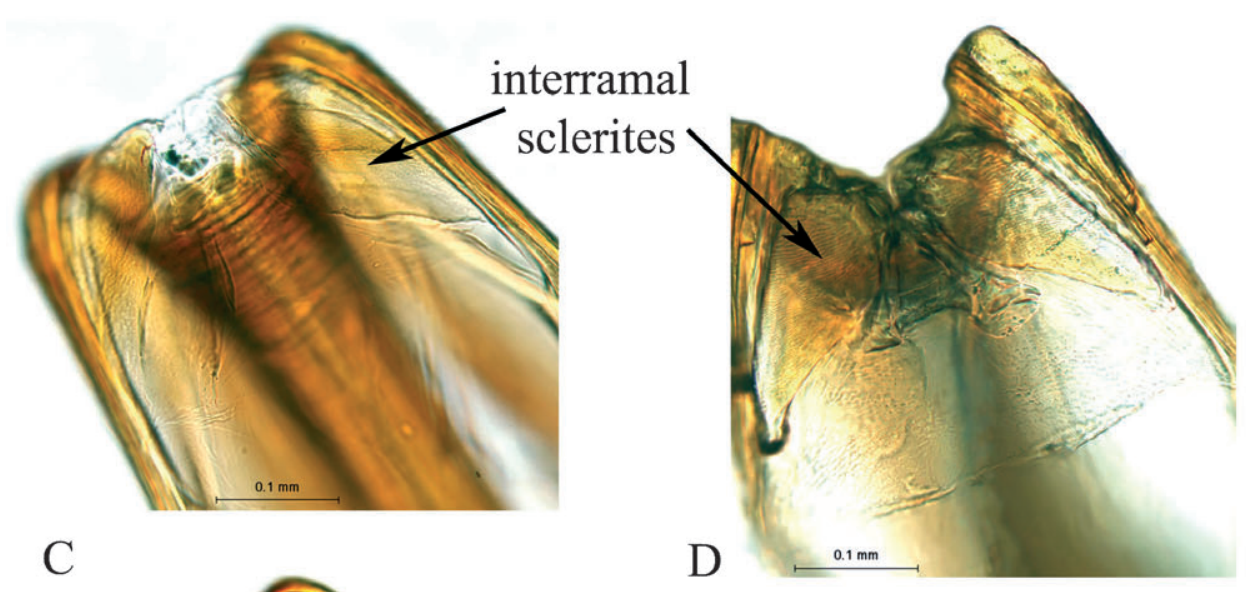

$\mathrm{E}$
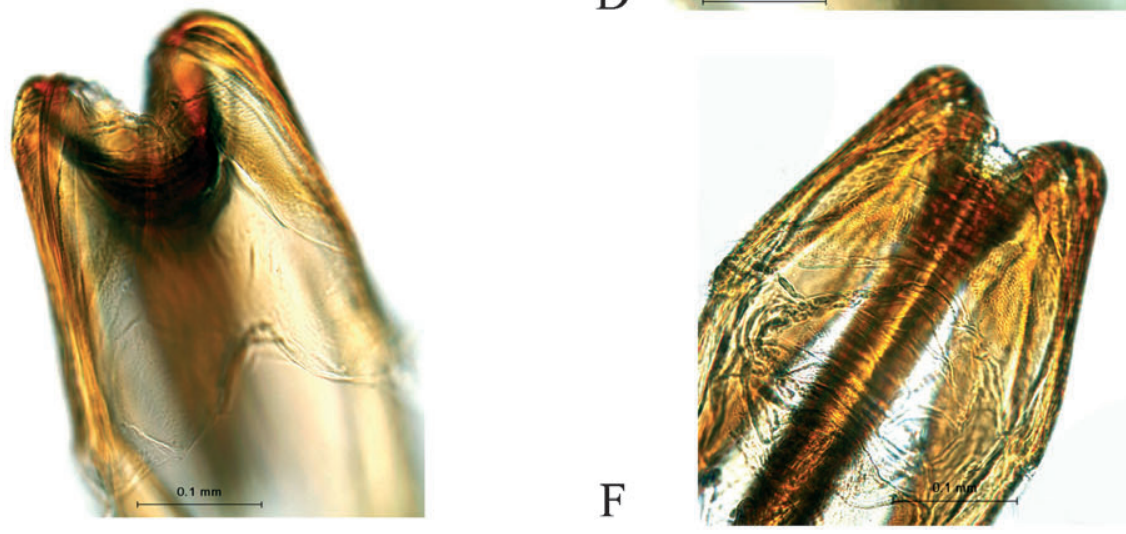

Fig. 18. Female genitalia of Psallus spp. Posterior wall, dorsal view. A - P. flavescens; B - P. roseoguttatus; C - P. loginovae; D - P. vittatus; E - P. tonnaichanus; F - P. michaili. Scale bars: $0.1 \mathrm{~mm}$.

(cat.); Yasunaga \& Vinokurov, 2000: 656 (key, fig.); Yasunaga, 2001: 173 (diag., fig.); Anufriev et al., 2001 (Engl. transl.); Kwon et al., 2001: 180 (cat.).

Diagnosis. Variously colored dorsum (dark brown to black), entirely pale antennae with dark base on segment I, completely black femora with pale apices (or tinged with red), pale tibiae with castaneous spots at the base of black spine (Fig. 6E-F). For a detail description see Josifov, 1983.

Male genitalia (Figs 9A-E): Endosoma C-shaped, laterally with numerous secondary processes, apex extends into short apical process, subapically extended membranous structure provided with numerous spinules (Figs
9A-B). Phallotheca illustrated as in Fig. 9C, left paramere as in Fig. 9D and right paramere as in Fig. 9E.

Female genitalia (Figs 13D, 16A): Bursa copulatrix moderate size, a membranous fold of dorsal labiate plate protrude medially as an inverted U-shaped curve, with central membranous wide fold extending from anterior to posterior portion of dorsal labiate plate, sclerotized ring elongate and narrow (Fig. 13D). Vestibular sclerites as in Fig. 16A.

Measurements. See Table 1.

Specimens examined. South Korea: Chuncheongnam-do: 10, Yesan-gun, Deoksan-myeon, Okgye, 4.vi.2007, light trap, J.W. Seong (SNU). Gangwon-do: 20, 5 $\$$, Donghae-si, Mt. Duta, 16.v.2001, light trap (NAAS); 10, Wonju-si, Munmak, 

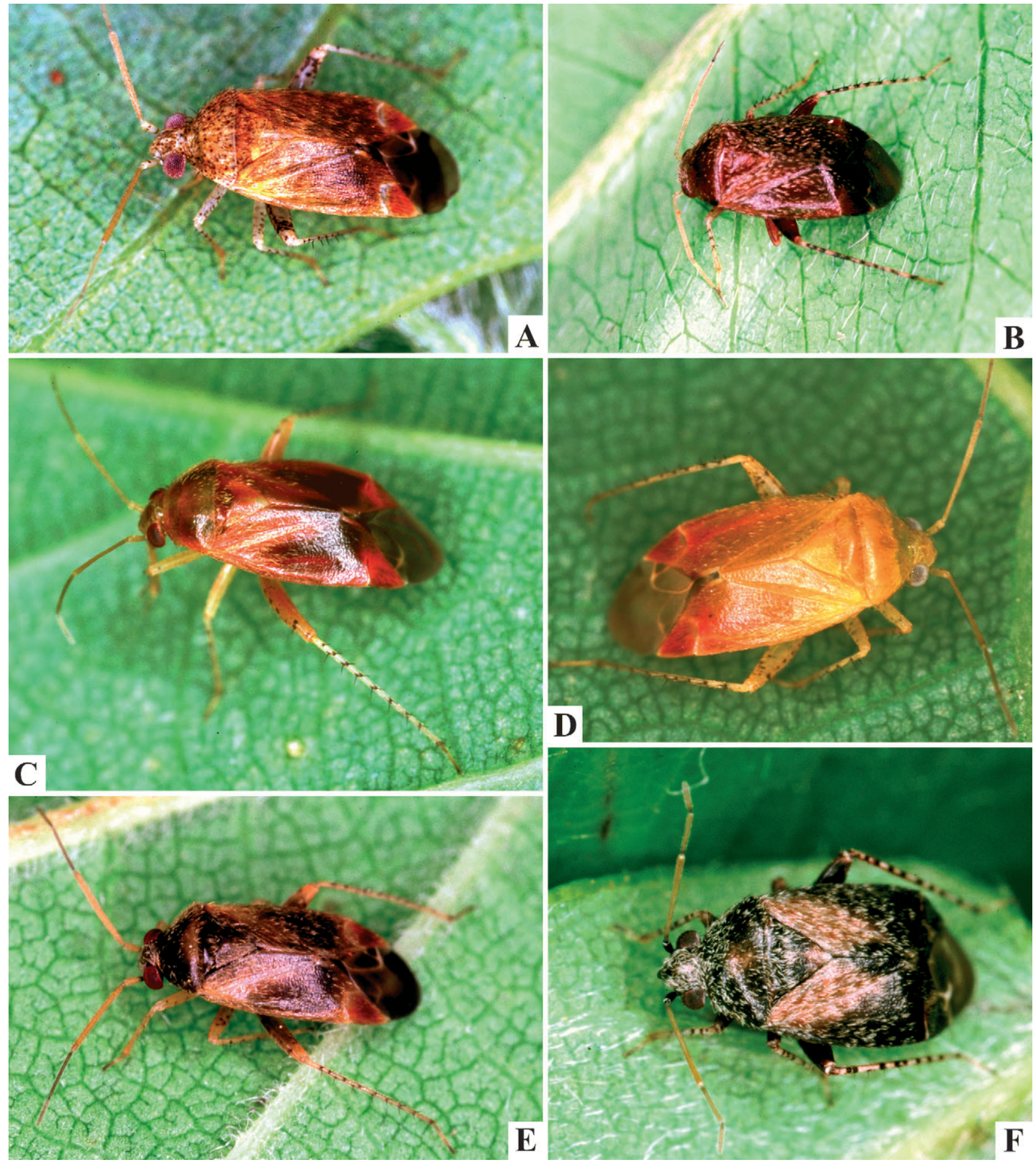

Fig. 19. Habitus images of live individuals of Psallus spp. A - P. roseoguttatus (from Shikoku, Japan); B - P. tonnaichanus (from Honshu, Japan); C - P. cinnabarinus (from Hokkaido, Japan); D - P. flavescens (from Hokkaido, Japan); E - P. ulmi (from Hokkaido, Japan; F - P. bagjonicus (from Kyushu, Japan). Photographed by M. Takai (A, F) and T. Yasunaga (B-E).

1.vi.2009, R.K. Duwal and S. Jung (AMNH_PBI 00383554) (SNU); Gyeonggi-do: 1 \%, Anyang-si, Gwanak Arboretum, 1.vi.2006, light trap, J.W. Seong (AMNH PBI 00383560) (SNU); 10 , Suwon, NAAS, 5.-8.vi.1997, light trap (SNU); 20 , Suwon-si, SNU Arboretum, 20.v.2009, R.K. Duwal (AMNH_PBI 00383558-383559) (SNU); 1, Suwon, 25.vi.2009, light trap, R.K. Duwal and S. Jung (SNU); 10 , 2 \% , Yangpyeong-gun, Mt. Yongmun, 11.vi.2009, light trap, R.K. Duwal and S. Jung (AMNH_PBI 00383561) (SNU); 20, 3 , same data except for date, 24.vi.2009 (SNU). Gyeongsangnam-do: 2\%, Sancheong-gun, Sicheon-myeon, 5.-6.vi.1997, S.B. Ann (SNU). Jeollanam-do: 20, Gwangyang-si, Choosan-ri, 16.-19.vi.2008, R.K. Duwal and S. Jung (SNU); 1 9 , Suncheon-si, Seungju-eup, Mt. Jogye, 24.vi.1997, S.B. Ann (SNU); 20ðે, 21 , Jangseong-gun, Mt. Bangjang, 24.vi.2010, light trap, R.K. Duwal (AMNH PBI 00383555; 383556-383557) (SNU). Japan: Shikoku: 1 ỗ, Kochi 
Pref., Ashizuri, 22.v.1999, M. Takai, determined by T. Yasunaga, 2008 (SNU).

Distribution. China, Japan (Honshu, Shikoku), Korea (North, South*).

Biology. This species is associated with fagaceous plants, Castanea (Josifov, 1983) and Quercus serrata (Yasunaga \& Vinokurov, 2000). However, the host in Korea is unknown as most of the specimens were collected in light.

\section{Psallus (Phylidea) cinnabarinus Kerzhner, 1979}

Figs 8A, 9F-J, 13E, 19C

Psallus (Phylidea) cinnabarinus Kerzhner, 1979: 44 (desc., figs); Kerzhner, 1988a: 847 (key, fig.); Schuh, 1995: 403 (cat.); Kerzhner \& Josifov, 1999: 405 (cat.); Yasunaga \& Vinokurov, 2000: 656 (key, fig.); Yasunaga, 2001: 174 (diag., fig.); Anufriev et al., 2001 (Engl. transl.).

Diagnosis. Deep red body, somewhat darker (or castaneous) head, pronotum, mesoscutum and scutellum, pale antennae and legs with distal two third of metafemora reddish, and castaneous red spots at the base of black spine on tibiae (Figs 8A, 19C). For a detail description see Kerzhner, 1979.

Male genitalia (Figs 9F-J): Endosoma S-shaped with broad apex extended into a short and slender apical process, margin of the apex covered with minute spinules (Figs 9G-H). Phallotheca illustrated as in Fig. 9F, left paramere as in Fig. 9I and right paramere as in Fig. 9J.

Female genitalia (Fig. 13E): Bursa copulatrix large, with wide seminal depository, membranous fold dorsal labiate plate divided into two halves medially and laterally furnished with scattered spinules, and sclerotized ring small oval and pointed anteriorly.

Measurements. See Table 1.

Specimens examined. South Korea*: Gangwon-do: 20 , Hongcheon-gun, 25.-26.vi.2003, light trap, J.W. Seong (AMNH_PBI 00383471-383472) (SNU). Gyeonggi-do: 10 , Gapyeong-gun, Mt. Yumyeong, 14.vi.1997, J.B. Ann (AMNH_PBI 00383473) (SNU); 3ठ, Yangpyeong-gun, Mt. Yongmun, 11.v.2009, light trap, R.K. Duwal and S. Jung (1 $\delta^{\star}$ in alcohol) (AMNH_PBI 00383474-383475) (SNU). Japan: Hokkaido: $1 \delta^{\star}$, Kasuga near Mt. Inoh Asahikawa C., 19.vii.1998, T. Yasunaga (AMNH_PBI 00383476) (SNU); 1 9 , 4-ban riv., Tobetsu, 10.vii.1991, H. Hiranuma, determined by T. Yasunaga, 2008 (AMNH_PBI 00383477) (SNU).

Distribution. Japan (Hokkaido), Korea (South)*, Russia (Sakhalin Is., Kurile Is.).

Biology. This species is associated with the elm trees (Ulmaceae), Ulmus propinqua (Kerzhner, 1978) and $U$. japonica (Yasunaga \& Vinokurov, 2000). However, in Korea the host is unknown as all the individuals were collected in light.

\section{Psallus (Phylidea) flavescens Kerzhner, 1988}

Figs 8B, 9K-N, 14A, 16C, 18A, 19D

Psallus (Phylidea) flavescens Kerzhner, 1988a (March): 847

(keys, figs); 1988b (April): 60 (species description, fig.); Schuh, 1995: 405 (cat.); Kerzhner \& Josifov, 1999: 406 (cat.); Yasunaga \& Vinokurov, 2000: 656 (key, distr., fig);
Yasunaga, 2001: 174 (diag., fig.); Anufriev et al., 2001: 126 (Engl. transl.).

Diagnosis. Entirely orange colored body, completely pale antennae and legs, base of cuneus, dark brown spots on metafemora basally arranged in two rows and irregularly scattered at apex, and brown spots on tibiae (Figs 8B, 19D). For a detail description see Kerzhner, 1988b.

Male genitalia (Figs 9K-N): Endosoma C-shaped, tubular, apical process arises from apex of secondary process, a short parallel membrane extends from apex and with minute spinules at the base, secondary gonopore located apically. Phallotheca illustrated as in Fig. 9N and left paramere as in Fig. 9M.

Female genitalia (Figs 14A, 16C, 18A): Bursa copulatrix of moderate size, seminal depositories wide, membranous folds on dorsal labiate plate furnished with dense spinules laterally, sclerotized rings small, oval but base somewhat straight and wide, and apex pointed (Fig. 14A). Vesitbular sclerites illustrated as in Fig. 16C and posterior wall as in Fig. 18A.

Measurements. See Table 1.

Specimens examined. South Korea*: Gwangwon-do: 20 , 4오. Hongcheon-gun, 25.-26.vi.2003, light trap, J.W. Seong and S. Jung (AMNH_PBI 00383478-383479; 00383480-383483) (SNU).

Distribution. China (North), Japan (Hokkaido, Honshu, Kyushu), Korea (South)*, Russia (Primorsk Terr.).

Biology. Yasunaga \& Vinokurov (2000) confirmed Tilia japonica (Tiliaceae) as a breeding host. In the Korean Peninsula the host plant is unknown, as all specimens were collected in light.

\section{Psallus (Phylidea) ernsti sp. n.}

Figs 6G-H, 9O-T, 15F

Holotype. North Korea: đิ, Bei Phjongjan, Mt. Tesong, 7.vi.1987, M. Josifov. Deposited at Tiroler Landesmuseum, Innsbruck, Austria (AMNH_PBI 00383429) (TLMF).

Diagnosis. Small, entirely castaneous black body, castaneous spots on the tibiae (Fig. 6G-H), membranous and flat apex of endosoma with a row of spinules apically (Figs 9P-R).

Description. Male. Body small, oval. Coloration (Fig. 6G-H): Generally reddish to reddish black, shiny; head, pronotum, mesoscutum and scutellum black; hemelytron, clavus and corium black or castaneous, membrane black. Venter uniformly black. Antennal segment I pale with reddish tinged and dark base, segment II pale, and segment III and IV brown. Labium, segment I-III castaneous and segment IV pale except at base. All coxae and trochanters castaneous; metafemora black with reddish apex, aggregation of black spots ventrally at the anterior margin; hind tibia pale with castaneous spots at the base of black spines. Surface and vestiture: Dorsum furnished with simple black setae and with uniformly distributed sericeous setae. Structure: Head: Projecting anteriorly; labium short, reaching apex of mesocoxae. Genitalia (Figs 9O-T): Endosoma: Shape more or less S-like, primary endosomal strap with a few long and short secondary processes, slightly curved elongated strap extends 
across secondary gonopore; secondary gonopore located subapically beneath a row of spinules. Phallotheca: Simple, not angulated, apex pointed. Left paramere: Body relatively short, anterior process short, flat and with blunt apex, posterior process comparatively short and slender. Right paramere: Body elongate, more or less parallel sided, apex with finger like process.

Female. Not significantly different from male in colour and vestiture, only the abdomen is slightly wider. Genitalia (Fig. 15F): Bursa copulatrix comparatively small, dorsal labiate plate furnished with spinules laterally; sclerotized rings small, ovate with somewhat narrow apex; the membranous fold on posterior of dorsal labiate plate supporting lateral oviducts at centre.

Measurements. See Table 1.

Specimens examined. Paratypes: North Korea: $1 \delta^{\hat{t}}$, same data as holotype except for date, 29.v.1975, M. Josifov (AMNH_PBI 00383430) (TLMF); $2 \%$, same data except date, 1.vi.1987, M. Josifov (AMNH_PBI 00383433, 383436) (TLMF); $2 \hat{\delta}$, same data except $\overline{\text { date, }}$ 7.vi.1987, M. Josifov (AMNH_PBI 00383431, 383435) (TLMF); 10, Bagjon, $20 \mathrm{~km}$ N Kaesong, 29.v.1987, M. Josifov (AMNH PBI 00383435) (SNU); 29, Phjongjang, Rjonggang, Mt. Rjongak, 31.v.1987, M. Josifov (AMNH_PBI 00383434, 383437) (TLMF, SNU).

Etymology. Named in honour of Dr. Ernst Heiss, for his outstanding contribution on the study of Heteroptera fauna.

Distribution. Korea (North)*.

Discussion. Morphologically very close to the species that occur on broad-leaf trees (e.g., oak or maple), like Psallus castaneae, P. tonnaichanus, P. kerzhneri and $P$. loginovae in color and vestiture. However, it is smaller than all these above mentioned species. Individuals of this species differ from $P$. tonnaichanus due to absence of a lateral tuft of bristles on the genital segment and separable from other three species by different structures of genitalia.

This new species is placed under subgenus Phylidea, as its endosoma is similar to that of the congeners, and unique serrated spinules on lateral margin.

The host plant for this species is unknown.

\section{Psallus (Phylidea) kerzhneri Josifov, 1992}

Figs 8C, 14B

Psallus (Phylidea) kerzhneri Josifov, 1992b: 115 (desc. figs); 1992a: 116 (list); Schuh, 1995: 408 (cat.); Kerzhner \& Josifov, 1999: 406 (cat.); Anufriev et al., 2001 (Engl. transl.); Kwon et al., 2001: 180 (cat.).

Diagnosis. Entirely black body tinged with red, black head, pronotum, mesoscutum and scutellum, reddish base of hemelytron darker towards the apex, reddish brown femora with pale apex and irregular black spots ventrally, and pale tibia with dark brown spots at the base of black spine (Fig. 8). For a detail description and illustrations of male genitalia see Josifov, 1992b: 115.

Female genitalia (Fig. 14B). Bursa copulatrix simple with asymmetrical large, elongated sclerotized rings laterally furnished with minute spinules.

Specimens examined. Paratypes: North Korea: 2 \% Bagjon, $20 \mathrm{~km} \mathrm{~N} \mathrm{Kaesong,} \mathrm{29.v.1987,} \mathrm{M.} \mathrm{Josifov,} \mathrm{determined} \mathrm{by} \mathrm{M.}$ Josifov (TLMF).

\section{Distribution. Korea (North).}

Biology. Josifov (1992b) documented the host plant of this species as Quercus dentata (Fagaceae).

\section{Psallus (Phylidea) loginovae Kerzhner, 1988}

Figs 8D-E, 11A-E, 14F, 16B, 18C

Psallus (Phylidea) loginovae Kerzhner, 1988a (March): 847 (key, fig.); 1988b (April): 59 (species description, fig.); Schuh, 1995: 408 (cat.); Kerzhner \& Josifov, 1999: 406 (cat.); Anufriev et al., 2001: 125 (Engl. transl.).

Diagnosis. Completely black head and pronotum, reddish or brownish hemelytron (in teneral individuals pale brown), entirely pale antennae with only the base of segment I dark, dirty yellow metafemora distally tinged with red, pale tibiae basally tinged with red and with castaneous or dark brown spots at the base of black spine (Fig. 8D-E). For a detail description see Kerzhner, 1988b.

Male genitalia (Figs 11A-E): Endosoma J-shaped with numerous short and long secondary processes, apex extended continuous to form apical process, a membranous structure arise at apex furnished marginally with spinules. Phallotheca illustrated as in Fig. 11A, left paramere as in Fig. 11B and right paramere as in Fig. 11C.

Female genitalia (Figs 14F, 16B, 18C): Bursa copulatrix of moderate size, dorsal labiate plate furnished with few scattered spinules, sclerotized rings strongly asymmetrical (Fig. 14F). Vestibular sclerites illustrated as in Fig. 16B and posterior wall as in Fig. 18C.

Measurements. See Table 1.

Specimens examined. South Korea*: Chungcheongbuk-do: 18 0 , 17\%, Danyan-gun, Mt. Sobaek (National Park), 25.-26.v.2009, Acer sp. (Aceraceae), R.K. Duwal and S. Jung (AMNH_PBI 00383524-383527; 383528-383532) (SNU).

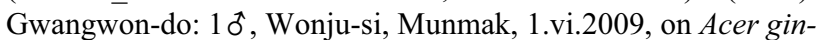
nala Maxim. (Aceraceae), R.K. Duwal and S. Jung (SNU). Gyeonggi-do: 30 , 5q, Panmunjom, 20.v.2008, S. Jung (AMNH_PBI 00383533-383534; 383535) (SNU).

Distribution. Korea (South)*, Russia (Primorsk Terr.).

Biology. Kerzhner (1988b) recorded the host plant of this species as Acer ginnala (Aceraceae), which was confirmed as breeding host in this study, as large numbers of nymphs were observed on this tree. This species appears at the end of May to early June in Korea. When collecting, we found it associated with a large numbers of plant hoppers, whose secretions made the tree completely sticky.

\section{Psallus (Phylidea) ulmi Kerzhner \& Josifov, 1966}

Figs $8 \mathrm{H}-\mathrm{K}, 19 \mathrm{E}$

Psallus (Phylidea) ulmi Kerzhner \& Josifov, 1966: 627 (desc. figs); Kerzhner, 1988a: 847 (key, fig.); Schuh, 1995: 416 (cat.); Kerzhner \& Josifov, 1999: 408 (cat.); Yasunaga \& Vinokurov, 2000: 657 (key, distr., fig.); Anufriev et al., 2001; (Engl. transl.); Kwon et al., 2001: 180 (cat.).

Diagnosis. Entirely red (or brown) body, pale antennae tinged with red on segment I and IV, black calli, pale base of cuneus, colour of femora similar to the dorsum (i.e. red femora in specimens with a red dorsum and brown femora in specimens with a brown dorsum), pale tibiae with small red spots (Figs 8H-K, 19E). For a detail 
description and structure of male genitalia see Kerzhner \& Josifov, 1966.

Specimens examined. Paratypes: Russia: $1 \hat{0}$, Burjatskaja, ASSR, Schara-gol am Flus Tschikoj, 1.vii.1928, (Lukjanovitsch) (AMNH PBI 00239157) (ZISP); same data as above, 1 ( (AMNH_PBI 00239162) (ZISP); 20, Amurgebiet, Blagovestschensk, 26.vi.1956, Kerzhner, determined by Kerzhner and Josifov (AMNH_PBI 00239108, 239109) (ZISP); 2 ㅇ, same data as above (AMNH PBI 00238967, 238968) (ZISP). Mongolia: $1 \delta^{\widehat{t}}$, Ulan-Bator, $40 \mathrm{~km}$ Ostl. von, 3.viii.1928 (A. Ivanov) (AMNH_PBI 00239146) (ZISP); 19 , same data as above, (AMNH_PBI 00238994) (ZISP).

Distribution. China (Northeast, North, Northwest), Japan (Hokkaido), Korea (North), Mongolia, Russia (Southeast, Far East).

Biology. This species is associated with elm (Ulmaceae), Ulmus pumila (Kerzhner \& Josifov, 1966) and Ulmus japonica, (Yasunaga \& Vinokurov, 2000)

\section{Subgenus Pityopsallus Wagner, 1952}

The subgenus Pityopsallus is a Holarctic group, comprised of seventeen species (Kerzhner \& Josifov, 1999; Yasunaga \& Vinokurov, 2000). The East Asian Pityopsallus was broadly revised by Vinokurov (1998) with nine species and confirmed that they inhabit conifers.

Members of this subgenus are recognized by dark dorsum, pale or brownish antennae, segment II as long as width of pronotum, labium surpassing apex of metacoxae (except in vittatus), pygophore with keel, apical process of endosoma elongated bearing teeth like spines laterally. For a detail description see Wagner, 1952.

\section{Psallus (Pityopsallus) kimi Josifov, 1983}

Fig. 10D-E

Psallus (Pityopsallus) lapponicus kimi Josifov, 1983: 210; 1992a: 116 (list).

Psallus (Pityopsallus) salicicola Schwartz \& Kelton, 1990: 941 (desc. figs).

Psallus Pityopsallus kimi Vinokurov, 1998: 285 (syn. n.); Schuh, 1999: 408 (cat.); Kerzhner \& Josifov, 1999 (cat.); Kwon et al., 2001: 181 (cat.).

Diagnosis. Brown (male) or yellowish brown (female) body, pale cuneus (somewhat whitish), dirty yellow or pale brown antennae with darker segment IV (Fig. 10D-E). For a detail description and structure of male genitalia see Josifov, 1983, or Vinokurov, 1998: 285.

Specimens examined. For reference: Russia: $1 \hat{\sigma}$, S. Yakutia, Chulmakan Rv. 577 m alt., 18 km NE of Chulman, 13.vii.1995, on Salix, T. Yasunaga, determined by F. Konstantinov, 2010 (AMNH_PBI 00240680) (ZISP); 1 \&, S. Yakutia, Nakhot 900 $\mathrm{m}, 17 \overline{\mathrm{km}}$ SE of Chulman, Mts Stanovoj, 11.vii.1995, on conifer, T. Yasunaga, determined by F. Konstantinov, 2010 (AMNH_PBI 00240683) (ZISP).

Distribution. Korea (North), Russia (Far East), Canada (North), USA (Alaska).

Biology. Psallus kimi and its sister species P. lapponicus, are easily confused, and the former has been synonymised with the later. Vinokurov (1998) declared kimi and lapponicus to be distinct species based on the length of the labium, which in P. lapponicus, it reaches or sur- passes middle of the abdomen while in $P$. kimi it reaches up to or slightly surpasses the metacoxae.

This species is recorded on Salix spp. (Salicaceae) by Josifov (1983) in North Korea whereas Yasunaga collected it on conifers in the Far East Russia.

\section{Psallus (Pityopsallus) luridus Reuter, 1878}

Figs 10A, 11F-J

Psallus luridus Reuter, 1878: 133.

Psallus Pityopsallus luridus: Kerzhner, 1978: 44 (list); 1988b: 848 (key, fig.); Schuh, 1995: 408 (cat.); Kerzhner \& Josifov, 1999: 409 (cat.); Anufriev et al., 2001: 127 (Engl. transl.); Kwon et al., 2001: 181 (cat.).

Diagnosis. Brown dorsum, much darker head, pronotum, mesoscutum and scutellum, entirely brown antennae and legs, dark brown spots ventrally on distal half of metafemora, and small brown spots on tibiae (Fig. 10A). For a detail description see Kerzhner, 1978.

Male genitalia (Figs 11F-J): Endosoma S-shaped, curved apex apically extended into an elongated apical process at the base of which posses minute spinules, a membrane arises between the apex and middle of the endosoma (Figs 11F-G). Phallotheca illustrated as in Fig. $11 \mathrm{H}$, left paramere as in Fig. $11 \mathrm{~J}$ and right paramere as in Fig. 11I.

Specimens examined. North Korea: $1 \hat{\delta}$, Jongkang-do, Samdzijon, 13.-19.vii.1974, on Larix sp. (Pinaceae), M. Josifov, determined by M. Josifov (SNU); 1 , Bosok-ri, $1000 \mathrm{~m}$, 20.vii.1974, on Larix sp. (Pinaceae), M. Josifov, determined by M. Josifov (SNU).

Distribution. China (Northeast), Korea (North), Russia (Sakhalin Is., Kurile Is.), Europe.

Biology. This species is associated with Larix sp. (Pinaceae) (Kerzhner, 1978).

\section{Psallus (Pityopsallus) vittatus (Fieber, 1861)}

Figs 10B-C, 11K-O, 15E, 17E, 18D

Agalliastes vittatus Fieber, 1861: 312.

Sthenarus roseri var. decolor Gredler, 1874: 557.

Psallus vittatus: Reuter, 1909: 75 (host); Kerzhner, 1988b: 847 (key, fig.); Schuh, 1995: 418 (cat.); Kerzhner \& Josifov, 1999: 410 (cat.); Anufriev et al., 2001: 127 (Engl. transl.); Kwon et al., 2001: 181 (cat.).

Diagnosis. Dark brown (or black) dorsum, black head, pronotum and scutellum, brown femora with pale apices and rows of spots on tibia obscure (Fig. 10B-C). For a detail description see Vinokurov, 1998.

Male genitalia (Figs 11K-O): Endosoma S-shaped with broad apex bearing an apical process along the margin of which there is a series of minute spinules. Phallotheca illustrated as in Fig. 11O, left paramere as in Fig. 11M and right paramere as in Fig. $11 \mathrm{~N}$.

Female genitalia (Figs 15E, 17E, 18D): Bursa copulatrix small, structures very delicate, membranous fold socket like, sclerotized rings very delicate or obscure (Fig. 15E). Vestibular sclerites illustrated as in Fig. 17E and posterior wall as in Fig. 18D.

Specimens examined. North Korea: $3 \delta^{\star}$, Bei Phjongjan, Mt. Taesong, 7.vi.1987, on Larix sp. (Pinaceae), M. Josifov, deter-

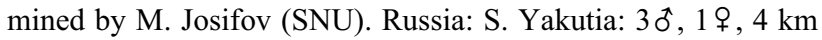


E of Nagornyj, Mt. Stanovoj, $800 \mathrm{~m}$ alt., 15.-16.vii.1995, on Larch sp. (Pinaceae), T. Yasunaga, determined by T. Yasunaga, 2008 (AMNH_PBI 00383498, 383500; 383499) (SNU).

Distribution. China (North), Korea (North), Russia (Sakhalin Is., Kurile Is.), Europe.

Biology. Kerzhner (1978) recorded the host plant of this species as Larix sp. (Pinaceae). Likewise the examined specimens in this study were collected by Josifov and Yasunaga from larch, Larix sp.

\section{Subgenus Psallus Fieber, 1858}

The subgenus Psallus is a large Holarctic group comprised of around fifty five species (Kerzhner \& Josifov, 1999; Yasunaga \& Josifov, 2000).

Members of this subgenus are variously coloured (bright, or dark, or pale) and have a short and sclerotized endosoma, with a few or numerous denticle like spines at the apex. For a detail description see Wagner, 1952.

\section{Psallus (Psallus) amoenus Josifov, 1983}

Figs $8 \mathrm{~F}-\mathrm{G}, 12 \mathrm{~A}-\mathrm{C}, 13 \mathrm{~B}, 17 \mathrm{C}$

Psallus (Psallus) amoenus Josifov, 1983: 208 (desc., figs); 1992a: 116 (list); Kerzhner, 1988: 845 (key, fig.); Schuh, 1995: 400 (cat.); Kerzhner \& Josifov, 1999: 411 (cat.); Anufriev et al., 2001: 123 (Engl. transl.); Kwon et al., 2001: 181 (cat.).

Diagnosis. Pale or yellowish body, with red speckles distributed on dorsum, brown spots restricted to anterior region of pronotum, completely pale antennae with basal ring of segment I brown, pale brown femora tinged with red and ventrally with large dark brown spots, and pale tibiae with small brown spots (Fig. 8F-G). For a detail description see Josifov, 1983.

Male genitalia (Figs 12A-C): Endosoma, see Josifov, 1983: 204. Phallotheca illustrated as in Fig. 12C, left paramere as in Fig. 12A and right paramere as in Fig. $12 \mathrm{~B}$.

Female genitalia (Figs 13B, 17C): Bursa copulatrix large, dorsal labiate plate furnished with numerous spinules and rectangular membranous fold anteriorly. Sclerotized rings wide and asymmetrical (Fig. 13B). Vestibular sclerites as in Fig. 17C.

Specimens examined. North Korea: $10,1 \%$, Bei Phjongjan, Mt. Tesong, 21.v.-1.vi.1987, on Quercus dentata Thunb. (Fagaceae), M. Josifov, determined by M. Josifov (SNU).

Distribution. Korea (North), Russia (Far East).

Biology. Josifov (1983) recorded the host plant of this species as Quercus dentata Thunb. (Fagaceae).

\section{Psallus (Psallus) bagjonicus Josifov, 1983}

Figs 10F-G, 12D-G, 13C, 16F, 19F

Psallus (Psallus) bagjonicus Josifov, 1983: 205 (desc., figs); 1992a: 116 (list); Schuh, 1995: 401 (cat.); Kerzhner \& Josifov, 1999: 412 (cat.); Yasunaga \& Vinokurov, 2000: 659 (key, figs); Yasunaga, 2001: 176 (diag., figs); Anufriev et al., 2001 (Engl. transl.); Kwon et al., 2001: 182 (cat.).

Diagnosis. Blackish (male) and brownish (female) body colouration, usually black head, pronotum and scutellum (in female with pale medial stripe that extends from head to pronotum and 3 pale spots on scutellum), brown metafemora with chain of dark spots, pale tibiae with a large castaneous spot at base of pale spine (Figs 10F-G, 19F). For a detail description see Josifov, 1983.

Male genitalia (Figs 12D-G): Endosoma C-shaped, apically provided with few elongated spines of various size and slender apical process (Figs 12F-G). Phallotheca illustrated as in Fig. 12D and left paramere as in Fig. 12E.

Female genitalia (Figs 13C, 16F): Bursa copulatrix of moderate size, dorsal labiate plate with a few scattered spinules and posterior region with somewhat rectangular membrane curved medially; sclerotized rings small, oval with broad base and slightly narrower apex (Fig. 13C). Vestibular sclerites as in Fig. 16F.

Measurements. See Table 1.

Specimens examined. South Korea: Gyeonggi-do: 1 , Gwangju-si, Mt. Taehwa, 2.-20.v.2008, Malaise trap, J.O. Lim (AMNH_PBI 00383494) (SNU); 2 \%, Osan-si, Sucheong-dong, 20.vi.1998, light trap, H.K. Lee (AMNH_PBI 00383492-383493) (SNU); 1 \%, Suwon-si, NĀAS, 10.-11.v.1997, light trap (NAAS). Japan: 10, 2 q, 6.v.1996, T. Yasunaga, determined by T. Yasunaga (AMNH_PBI 00383490; 383491) (SNU).

Distribution. Korea (North, South*), Japan (Kyushu, Shikoku).

Biology. This species is associated with oak (Fagaceae), Quercus aliena Blume, (Josifov, 1983) in North Korea, and Q. serrata Murray and Q. acutissima Carruth (Yasunaga \& Vinokurov, 2000) in Japan. However, the host is unknown in South Korea, as all specimens were collected in light and Malaise traps.

\section{Psallus (Psallus) cheongtaensis sp. n.}

Figs $10 \mathrm{H}, 12 \mathrm{~J}-\mathrm{N}$

Holotype. South Korea*: Gwangwon-do: 10 , Hoengseong-gun, Dunnae-myeon, Mt. Cheongtae (Natural recreation forest), 20.-21.vii.2000, H.G. Goh. Deposited at National Academy of Agricultural Science (AMNH_PBI 00383428) (NAAS).

Diagnosis. Entirely pale (somewhat pale yellowish) body and appendages, uniformly distributed simple semierect dark setae on dorsum, large brown spots at the base of black spines on hind tibia (Fig. 10H), a pair of protuberances on either side of genital segment (Fig. $12 \mathrm{H}$ ) and a pair of ear like secondary processes apically on endosoma and medially developed elongated flat apical process (Figs 12J-L).

Description. Male. Body elongate oval. Coloration (Fig. 10H): Body uniformly pale dorsally and ventrally (or somewhat pale yellowish in dry specimens). Antennae pale, segments III and IV pale brown. Labium, segment I and II pale, segment III brown and segment IV dark brown distally. Legs pale; metafemora ventrally with a row of spots on anterior margin, few fused spots scattered on the base and numerous irregular spots on distal half; tibiae pale with rows of large brown spots at the base of black spines; membrane greyish. Surface and vestiture: Dorsum furnished with uniformly distributed simple black semierect setae and sericeous setae. Structure: Head: Length short compare to body size; inter-ocular space wide; labium slightly surpassing apex of mesocoxae. Genitalia (Fig. 12H-N): Endosoma: Shape C-like; 
apex with a pair of ear like secondary processes arising from each side, and a medially developed elongated flat apical process; secondary gonopore large and positioned subapically. Phallotheca: As in Fig. 12M. Left paramere: Body large, both anterior and posterior processes flat. Right paramere: Body elongate, subapically furnished with notch like outgrowth and apex tapered to form a short finger like process.

Measurements. See Table 1.

Specimens examined. Known from the holotype only.

Etymology. Named after the type locality, Mt. Cheongtae.

Distribution. Korea (South)*.

Discussion. This new species is superficially very similar to Europiella gilva (in terms of coloration and spots on femora). However, the male genitalia is unlike those of the Europiella species and similar to that of the Psallus species treated herein.

This new species is described under subgenus Psallus, due to its similar appearance of endosoma to that of the other congeners, and a broad apex with numerous short and flat elongated processes.

The biology is unknown.

\section{Psallus (Psallus) koreanus Josifov, 1983}

Fig. 10I-J

Psallus (Psallus) koreanus Josifov, 1983: 209 (desc., figs).

Psallus koreanus: Kerzhner, 1988a: 847 (key); Josifov, 1992a: 116 (list); Schuh, 1995: 408 (cat.); Kerzhner \& Josifov, 1999:

415 (cat.); Anufriev et al., 2001: 126 (Engl. transl.); Kwon et al., 2001: 182 (cat.).

Diagnosis. Deep red body, pale vertex, shiny blackish pronotum, mesoscutum, scutellum and base of hemelytron (or uniformly brownish red), deep red cuneus, and red metafemora with black spots on anterior margin (Fig. 10I-J). For a detail description and structures of male genitalia see Josifov, 1983: 209.

Specimens examined. Paratypes: North Korea: $10,1 \%$, Bagjon, $20 \mathrm{~km}$ N Kaesong, 21.-23.v.1975, M. Josifov, determined by M. Josifov (AMNH_PBI 00240712; 240714) (ZISP).

Distribution. Korea (North), Russia (Far East).

Biology. This species is recorded on various rosaceous trees like, e.g., Crataegus sp., Prunus sp., and Sorbus amurensis, and also observed on Picea sp. (Pinaceae) (Josifov, 1983).

\section{Psallus (Psallus) sanguinarius Kerzhner \& Josifov, 1999}

Psallus (Psallus) sanguinolentus Josifov, 1983: 209 (junior primary homonym of Psallus graminicola f. sanguinolenta Stichel, 1956); Schuh, 1995: 415 (cat.).

Psallus sanguinarius Kerzhner \& Josifov, 1999: 417 (new name, cat.); Kwon et al., 2001: 183 (cat.).

Diagnosis. Brownish red dorsum; ocher head, dark markings on fore-head, black brown clypeus and pale antennae. For a detail description see Josifov, 1983 (where it is described as sanguinolentus).

Distribution. Korea (North).

Note. Josifov (1983) recorded this species on Sorbus amurensis (Rosaceae) and on Picea (Pinaceae) in North
Korea. We were unable to observe any specimens for this study.

ACKNOWLEDGEMENTS. We are very thankful to the late M. Josifov, for his contribution to the knowledge and collection of Psallus from North Korea. Our sincere gratitude goes to E. Heiss (TLMF) for arranging the loan specimens and his encouragements and suggestions. Special thanks are extended to M.D. Schwartz (Agriculture Canada, Ottawa) and D. Wyniger (NaturMuseum Luzern, Switzerland) for reviewing and invaluable comments on the manuscript. The first author appreciates the efforts of G. Lee, (NAAS), F.V. Konstantinov and V.M. Genezdilov (ZISP) in providing their collections for this study and arranging loan of specimens, P. Štys (Charles University, Czech Republic) for his kind advice and A.F.G. Dixon for editing language corrections. Thanks to J. Lim, S. Kim, M. Kim, H. Choi, S. Shin (Insect Biosystematics Laboratory, SNU) for help during field trips and M. Takai (Kochi, Japan) was kind enough to provide digital images of $P$. roseoguttatus and P. bagjonicus. This research was supported by grants from the Agenda Project (Grant No. 102966290) of the Rural Development Administration in the Republic of Korea, the Basic Science Research Program of the National Research Foundation of Korea (NRF) funded by the Ministry of Education, Science and Technology (2011-0011055), and "The Survey of Korean Indigenous Species" supported by National Institute of Biological Resources (NIBR) of Ministry of Environment of Korea.

\section{REFERENCES}

Anufriev G.A., Danzing E.M., Emeljanov A.F., Golub V.B., Kanyukova E.V., Kerzhner I.M., Konovalova Z.A., Pashchenko N.F., Tshernova G.P. \& Vinokurov N.N. 2001: Keys to the Insects of the Far East of the USSR. Vol. 2. U.S. Department of Agriculture, $211 \mathrm{pp}$. [English translation].

CAssis G. 2008: The Lattinova complex of Austromirine plant bugs (Hemiptera: Heteroptera: Orthotylinae). Proc. Entomol. Soc. Wash. 110: 845-939.

DAvis T.N. 1955: Morphology of the female organs of reproduction in the Miridae (Hemiptera). Ann. Entomol. Soc. Am. 48: 132-150.

FALLÉN C.F. 1826: Supplementum Cimicidum Sueciae. Berling, Londini Gothorum [= Lund], 16 pp.

FIEBER F.X. 1858: Criterien zur generischen Theilung der Phytocoriden (Capsini aut.). Wien. Entomol. Monatschr. 2: 289-327, 329-347, 388.

FIEBER F.X. 1861: Die Europäischen Hemiptera. Halbflügler. (Rhynchota, Heteroptera). Gerold's Sohn, Wien, vi +444 pp.

GredLER P.V.M. 1874: Nachlese zu den Wanzen Tirols. Verh. Zool.-Bot. Gesell. Wien 24: 553-558.

Henry T.J. \& Wheeler A.G. JR. 1979: Palearctic Miridae in North America: records of newly discovered and little-known species (Hemiptera: Heteroptera). Proc. Entomol. Soc. Wash. 81: $257-268$

Josifov M. 1983: Neue Psallus-Arten aus Nord-Korea (Heteroptera, Miridae). Reichenbachia 21: 197-211.

Josifov M. 1992a: Contribution to a knowledge of the family Miridae of North Korea (Heteroptera). Insecta Kor. 9: $115-128$.

Josifov M. 1992b: Neue Miriden aus Korea (Insecta, Heteroptera). Reichenbachia 29: 105-118.

KERZHNER I.M. 1962: Materials on the taxonomy of capsid bugs (Hemiptera, Miridae) in the fauna of the USSR. Entomol. Obozr. 41: 371-382 [in Russian]. 
KeRZHNER I.M. 1973: Heteroptera of the Tuvinian ASSR. Trudy Biol. Inst. Sibir. Otd. Akad. Nauk SSSR (Novosibirsk) 16: 78-92 [in Russian].

KerzhNeR I.M. 1978: Bugs (Heteroptera) of Sakhalin and the Kuril Islands. Trudy Biologopochv. Inst. Dal'nevost. Otdel. Akad. Nauk SSSR (N.S.) 50: 31-57 [in Russian].

KERZHNER I.M. 1979: New Heteroptera from the Far East of the USSR. Trudy Zool. Inst. Akad. Nauk SSSR 81: 14-65 [in Russian].

KerZHNER I.M. 1988a: Family Miridae. In Lehr P.A. (ed.): Keys to the Insects of the Far East of the USSR. Vol. 2. Nauka, Leningrad, pp. 778-857 [in Russian].

Kerzhner I.M. 1988b: New and Little-known Heteropterous Insects (Heteroptera) from the Far East of the USSR. Acad. Sci. USSR, Far Eastern Centre, Vladivostok, (1987) 84 pp. [in Russian].

KeRZHNer I.M. \& Josifov M. 1966: Beschreibung neuer Arten von Landwanzen (Heteroptera) aus der Mongolischen Volksrepublik und Bemerkungen über Phytocoris turkestanicus Pop. Bull. Acad. Polon. Sci. 14: 627-634.

Kerzhner I.M. \& Josifov M. 1999: Catalogue of the Heteroptera of the Palearctic Region. The Netherlands Entomological Society, Ponsen and Looijen, Wageningen, xiv +577 pp.

KERZHNER I.M. \& SCHUH R.T. 1995: Homonymy, synonymy, and new combinations in the Miridae (Heteroptera). Am. Mus. Nov. 3137: 1-11.

KULIK S.A. 1965: Blindwanzen Ost-Sibiriens und des Fernen Ostens (Heteroptera - Miridae). Acta Faun. Entomol. Mus. Natn. Pragae 11: 39-69 [in Russian].

Kwon Y.J., SuH S.J. \& KIM J.A. 2001: Economic insects of Korea 18. Hemiptera. Insecta Kor. (Suppl.) 25: 513 pp.

LinNAVUORI R.E. 1993: The Phylinae (Hemipera: Miridae) of West, Central and North East Africa. Garcia de Orta (Sér. Zool.) 18[1991]: 115-296.

Muramoto N. 1973: A chromosome study in eighteen Japanese Heteropterans. La Kromosomo 91: 2896-2905.

Müller O.F. 1776: Zoologiae Danicae prodromus, seu animalium Daniae et Norvegiae indigenarum characteres, nomina et synonyma imprimis popularium. Hallager, Hafniae, xxxii $+282 \mathrm{pp}$.

ReUter O.M. 1878: Hemiptera Gymnocerata Europae. Hémiptères Gymnocérates d'Europe, du bassin de la Méditerranée et de l'Asie Russe. Vol. 1. Helsingfors, 188 pp.

ReUter O.M. 1883: Hemiptera Gymnocerata Europae. Hémiptères Gymnocérates d'Europe, du bassin de la Méditerranée et de l'Asie Russe. Vol. 3. Helsingfors, pp. 313-496.

ReUTER O.M. 1899: Capsidae novae mediterranae, descriptae. I. Öfversigt af Finska Vetenskaps-societetens Förhandlingar (B) 12: $131-162$.

ReUTER O.M. 1909: Bemerkungen über nearktische Capsiden nebst Beschreibung neuer Arten. Acta Soc. Sci. Fenn. 36(2): $1-86$

Reiger C. \& RABItsch W. 2006: Taxonomy and distribution of Psallus betuleti (Fallén) and P. montanus Josifov stat. nov. (Heteroptera: Miridae). Tijdschr. Entomol. 149: 161-166.

Rizzotтi V. 2000: The specific validity of Psallus pseudoambiguus Wagner, 1970. Boll. Soc. Entomol. Ital. 132: 17-21.

Sснин R.T. 1995: Plant Bugs of the World (Insecta: Heteroptera: Miridae): Systematic Catalog, Distributions, Host List, and Bibliography. New York Entomological Society, xii +1329 pp.
Schun R.T. 2006: Revision of phylogenetic, biogeographic, and host analysis of the endemic western North American Phymatopsallus group, with the description of 9 new genera and 15 new species (Insecta, Hemiptera, Miridae, Phylinae). Bull. Am. Mus. Nat. Hist. 301: 1-115.

Schun R.T. \& Wu G. 2009: Review of Eminoculus Schuh (Heteroptera: Miridae: Phylinae) from South Africa, with the description of five new species. Entomol. Am. 115: 36-66.

Schwartz M.D. \& Kelton L.A. 1990: Psallus salicicola, a new species, with additional records of recently discovered Palearctic Psallus Fieber from Canada (Heteroptera: Miridae: Phylinae). Can. Entomol. 122: 941-947.

SEIDENSTÜCKER G. 1962: Über einige Miriden aus Kleinasien mit Beschreibung von zwei neuen Halticinen (Heteroptera). Reichenbachia 1: 129-143.

TAKSDAL G. 1983: Orthotylus marginalis Reuter and Psallus ambiguus (Fallén) (Heteroptera, Miridae) causing stongy pits in pears. Acta Agric. Scand. 33: 205-208.

VinOKUROV N.N. 1998: Asian plant bugs of the subgenus Pityopsallus E. Wagn., genus Psallus Fieb. (Heteroptera: Miridae). Zoosyst. Rossica 7: 285-296.

WAGNER E. 1952: Zur Systematik von Psallus variabilis Fall. (Hem. Het. Miridae). Opusc. Entomol. 17: 90-94.

Wagner E. 1970: Vier neue Psallus-Arten aus Südeuropa (Hemiptera, Heteroptera, Miridae). Reichenbachia 12: 295-303.

Wagner E. 1975: Psallus (s. str.) amitinus n. sp. (Hemiptera, Heteroptera, Miridae). Reichenbachia 15: 303-306.

Wagner E. \& Weber H.H. 1964: Hétéroptères Miridae. Faune de France 67. Fédération Française des Sociétés de Sciences Naturelles, Paris, 591 pp.

Wheeler A.G. JR. 2001: Biology of the Plant Bugs (Hemiptera: Miridae). Cornell University Press, Ithaca and London, 507 pp.

Wheeler A.G. JR. \& Henry T.J. 1992: A Synthesis of the Holarctic Miridae (Heteroptera): Distribution, Biology and Origin, with Emphasis on North America. Entomological Society of America, Lanham, MA, $282 \mathrm{pp}$.

Wyniger D. 2006: The Central European Hallodapini: Studies of the female genitalia (Heteroptera, Phylinae, Miridae). Denisia 19: 711-720.

Yasunaga T. 2001: Family Miridae Hahn, plant bugs. In Yasunaga T., Takai M. \& Kawasawa T. (eds): A Field Guide to Japanese Bugs II. Zenkoku Noson Kyoiku Kyokai, Tokyo, pp. 1-96, 111-351 [in Japanese].

Yasunaga T. 2010: Plant bugs of the tribe Phylini in Thailand (Heteroptera: Miridae: Phylinae), with description of six new species from additional areas in Tropical and Subtropical Asia. Entomol. Am. 116(3/4): 50-92.

Yasunaga T. \& Schwartz M.D. 2007: Revision of the mirine plant bug genus Philostephanus Distant and allies (Heteroptera: Miridae: Mirini). Tijdschr. Entomol. 150: 101-180.

Yasunaga T. \& VinOKurov N.N. 2000: The phyline plant bug genus Psallus Fieber in Japan (Heteroptera: Miridae: Phylinae). Entomol. Sci. 3: 653-668.

ZaitZeva I.F. 1968: A review of the genus Psallus Fieb. (Heteroptera) from the Caucasus. Entomol. Obozr. 47: 865-877 [in Russian].

Zetterstedt J.W. 1838: Insecta Lapponica descripta. Vol. 1. Lipsiae, 868 pp.

ZHENG L.Y. \& Li H.Y. 1990: Four new species of Psallus Fieb. from China (Insecta, Hemiptera, Heteroptera: Miridae). Reichenbachia 28: 15-19.

Received December 9, 2010; revised and accepted February 13, 2012 